\title{
Structure and dynamics of the Martian lower and middle atmosphere as observed by the Mars Climate Sounder: Seasonal variations in zonal mean temperature, dust, and water ice aerosols
}

\author{
D. J. McCleese, ${ }^{1}$ N. G. Heavens, ${ }^{2}$ J. T. Schofield, ${ }^{1}$ W. A. Abdou, ${ }^{1}$ J. L. Bandfield, ${ }^{3}$ \\ S. B. Calcutt, ${ }^{4}$ P. G. J. Irwin, ${ }^{4}$ D. M. Kass, ${ }^{1}$ A. Kleinböhl, ${ }^{1}$ S. R. Lewis, ${ }^{5}$ D. A. Paige,${ }^{6}$ \\ P. L. Read, ${ }^{4}$ M. I. Richardson, ${ }^{7}$ J. H. Shirley, ${ }^{1}$ F. W. Taylor, ${ }^{4}$ N. Teanby, ${ }^{4}$ \\ and R. W. Zurek ${ }^{1}$
}

Received 14 June 2010; revised 3 September 2010; accepted 28 September 2010; published 28 December 2010.

[1] The first Martian year and a half of observations by the Mars Climate Sounder aboard the Mars Reconnaissance Orbiter has revealed new details of the thermal structure and distributions of dust and water ice in the atmosphere. The Martian atmosphere is shown in the observations by the Mars Climate Sounder to vary seasonally between two modes: a symmetrical equinoctial structure with middle atmosphere polar warming and a solstitial structure with an intense middle atmosphere polar warming overlying a deep winter polar vortex. The dust distribution, in particular, is more complex than appreciated before the advent of these high $(\sim 5 \mathrm{~km})$ vertical resolution observations, which extend from near the surface to above $80 \mathrm{~km}$ and yield 13 dayside and 13 nightside pole-to-pole cross sections each day. Among the new features noted is a persistent maximum in dust mass mixing ratio at $15-25 \mathrm{~km}$ above the surface (at least on the nightside) during northern spring and summer. The water ice distribution is very sensitive to the diurnal and seasonal variation of temperature and is a good tracer of the vertically propagating tide.

Citation: McCleese, D. J., et al. (2010), Structure and dynamics of the Martian lower and middle atmosphere as observed by the Mars Climate Sounder: Seasonal variations in zonal mean temperature, dust, and water ice aerosols, J. Geophys. Res., 115, E12016, doi:10.1029/2010JE003677.

\section{Introduction}

[2] The Mars Climate Sounder (MCS) [McCleese et al., 2007] on Mars Reconnaissance Orbiter (MRO) [Zurek and Smrekar, 2007] has been observing the Martian atmosphere since September of 2006. MCS observes the limb of the atmosphere in one broadband visible and eight infrared channels in the 0.3 to $50 \mu \mathrm{m}$ spectral range. MCS data can be used to determine the thermal structure of the atmosphere from the surface to $80 \mathrm{~km}$ at a vertical resolution of $\sim 5 \mathrm{~km}$ (approximately one half the atmospheric scale height). Thus,

\footnotetext{
${ }^{1}$ Jet Propulsion Laboratory, California Institute of Technology, Pasadena, California, USA.

${ }^{2}$ Division of Geological and Planetary Sciences, California Institute of Technology, Pasadena, California, USA.

${ }^{3}$ Department of Atmospheric Sciences, University of Washington, Seattle, Washington, USA.

${ }^{4}$ Department of Physics, University of Oxford, Oxford, UK.

${ }^{5}$ Department of Physics and Astronomy, Open University, Milton Keynes, UK.

${ }^{6}$ Department of Earth and Space Sciences, University of California, Los Angeles, California, USA.

${ }^{7}$ Ashima Research, Pasadena, California, USA.

Copyright 2010 by the American Geophysical Union. 0148-0227/10/2010JE003677
}

MCS bridges the gap between observations of the lower atmosphere provided by nadir infrared spectroscopy [Smith et al., 2001; Smith, 2004], from which an extensive climatology has been developed, and radio occultation [Hinson et al., 2004], and measurements in the upper atmosphere obtained from aerobraking experiments [Keating et al., 1998], and stellar occultation [Forget et al., 2009]. MCS data is also used to simultaneously profile the aerosol structure of the atmosphere with the same vertical range and resolution as its temperature observations.

[3] Initial retrievals from MCS data have been used to investigate the temperature maximum in the polar middle atmosphere during southern hemisphere winter [McCleese et al., 2008] and the atmospheric thermal tides [Lee et al., 2009]. Today, there are over $10^{6}$ MCS profiles of temperature, dust, and water ice [Kleinböhl et al., 2009] spanning the full seasonal cycle with some seasons sampled over multiple years.

[4] In this paper, we describe the seasonal cycle of the zonal average thermal structure and aerosol distributions during the first 1.5 Mars years of observations by MCS. We focus on Mars Year (MY) 29 (see Clancy et al. [2000] for a description of the convention), for which we have nearly complete observational coverage and during which there 


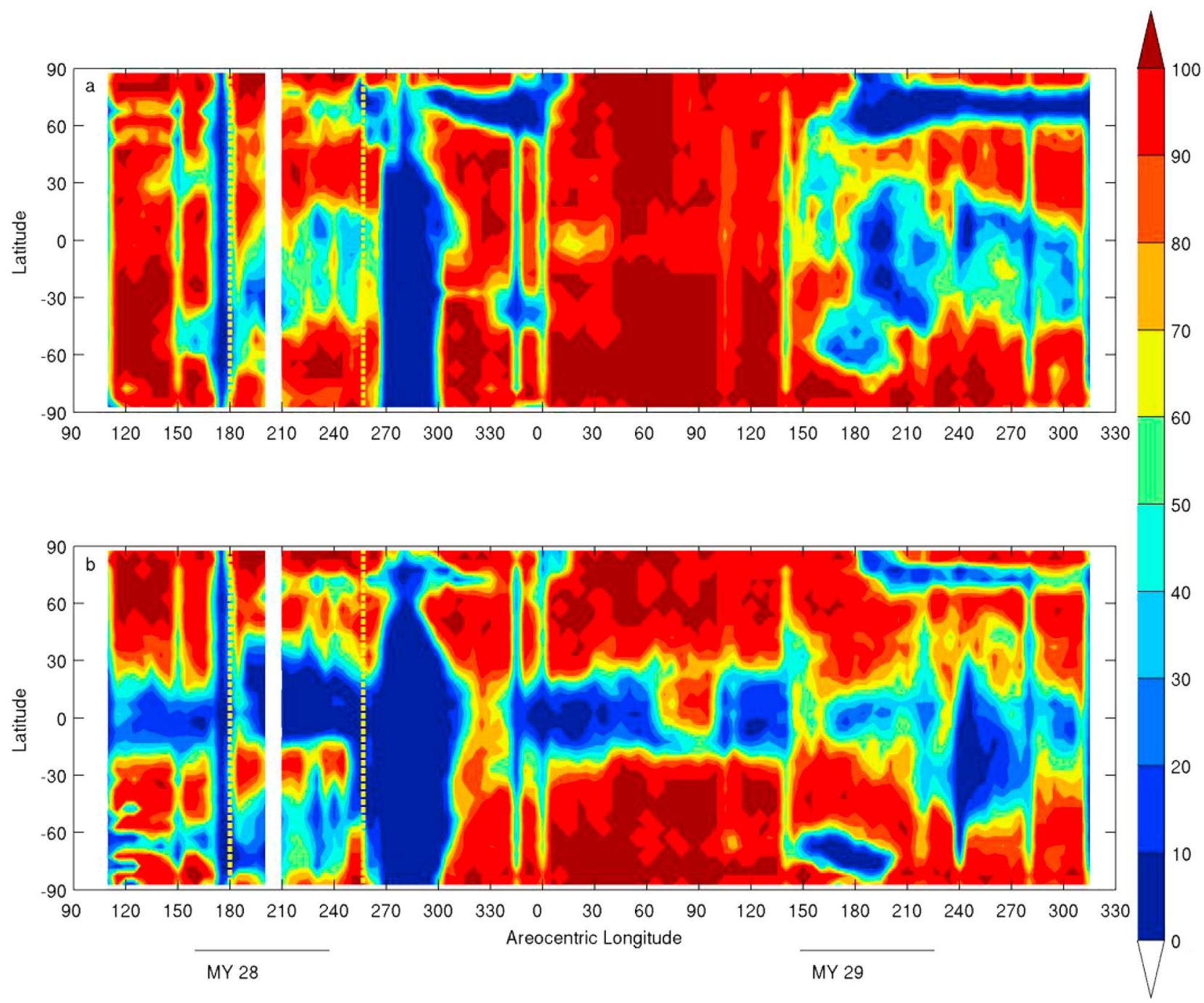

Figure 1. Percentage of longitude bins with successful MCS retrievals for each $\mathrm{L}_{\mathrm{s}} /$ latitudinal bin. The dashed yellow line denotes the period of limb staring: (a) nightside; (b) dayside. Contours are shown every $10 \%$.

was no global dust storm. We describe the data set used and its analysis, and provide an overview of seasonal variability in the thermal structure of the atmosphere, as well as the vertical distribution of dust and water ice. In a later paper, Heavens et al. (manuscript in preparation, 2010) focus on the implications of these observations for the mean meridional circulations of the lower and middle atmosphere and the degree to which these circulations are coupled.

\section{Nature of the Data and Retrievals}

\subsection{Retrieved Quantities}

[5] McCleese et al. [2007] describe the MCS instrument and observing strategy. Kleinböhl et al. [2009] provide an in-depth description of the first generation retrieval algorithm. At present, atmospheric retrievals from MCS measurements of radiance provide vertical profiles with respect to pressure, $p(\mathrm{~Pa})$, of temperature, $T(\mathrm{~K})$, dust opacity, i.e., the extinction per unit height due to dust, $d_{z} \tau_{d u s t}$ $\left(\mathrm{km}^{-1}\right)$ at $463 \mathrm{~cm}^{-1}$, and water ice opacity $d_{z} \tau_{H_{2} \text { Oice }}\left(\mathrm{km}^{-1}\right)$ at $843 \mathrm{~cm}^{-1}$. In addition, all retrievals include estimates of error for each quantity on each pressure level based on both the uncertainty in the observed radiances due to detector noise and the residuals in the fit to the observed radiances by the retrieval.

\subsection{Zonal Averaging and Sampling}

[6] MCS retrievals and quantities derived from them (as described in later in this section) are averaged after being binned by $\mathrm{MY}, \mathrm{L}_{\mathrm{s}}\left(5^{\circ}\right.$ resolution centered at $0^{\circ}, 5^{\circ}$ etc.); time of day: "dayside" (0900-2100 LST) and "nightside" (2100-0900 LST); scene latitude ( $5^{\circ}$ resolution); and scene longitude $\left(5.625^{\circ}\right.$ resolution). The spatial resolution of the binning is chosen to be comparable to standard Mars general circulation model grids. Mean latitude and longitude refer to the coordinates at the tangent point of the limb path as seen at the center of the MCS detector array, or about $40 \mathrm{~km}$ above the surface. Zonal averages are the average of all longitudinal bins containing data. 

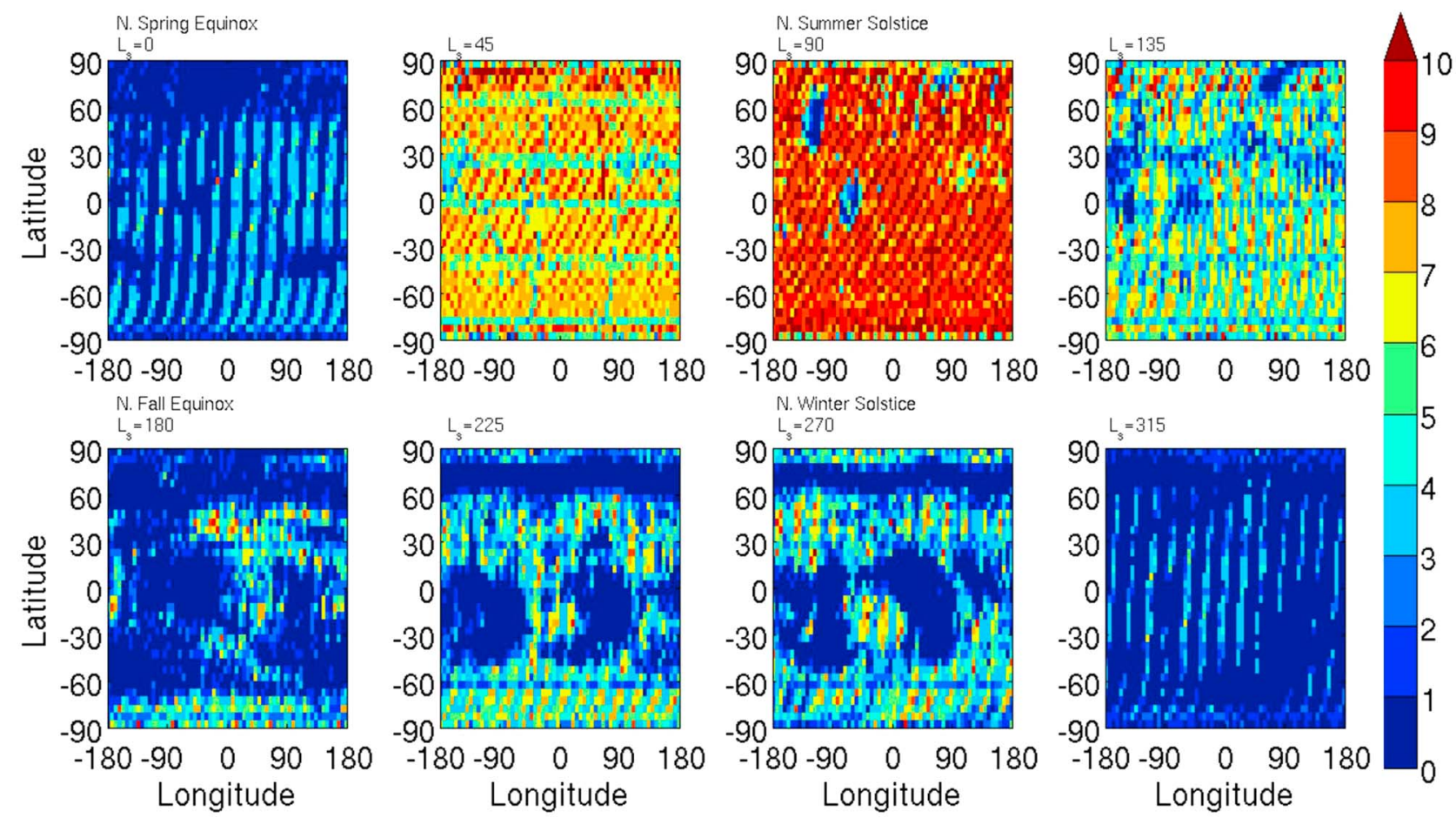

Figure 2. Number of retrievals per bin: nightside retrievals for the $\mathrm{L}_{\mathrm{s}}$ bins labeled at the top of each panel. The color scale is deepest red for 10 retrievals or more. For $\mathrm{L}_{\mathrm{s}}=0^{\circ}$, the strong striping pattern is consequence of operational conditions in which MCS was not collecting data for all but $\sim 3$ days. Horizontal striping, especially apparent in $\mathrm{L}_{\mathrm{s}}=45^{\circ}$ and $180^{\circ}$, is due to routine calibration sequences.
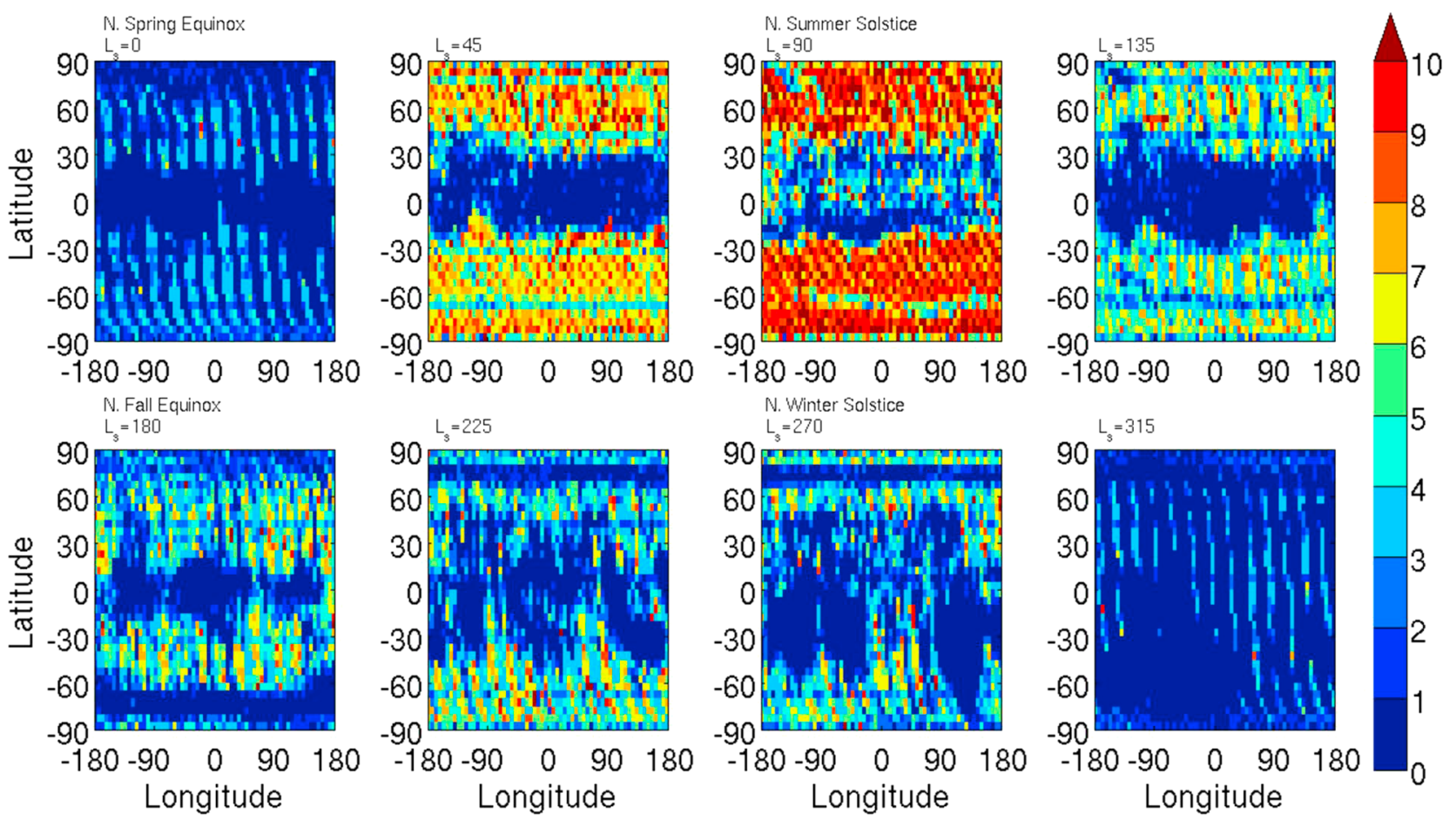

Figure 3. Number of retrievals per bin: dayside retrievals for the $\mathrm{L}_{\mathrm{s}}$ bins labeled at the top of each panel. The color scale is deepest red for 10 retrievals or more. 

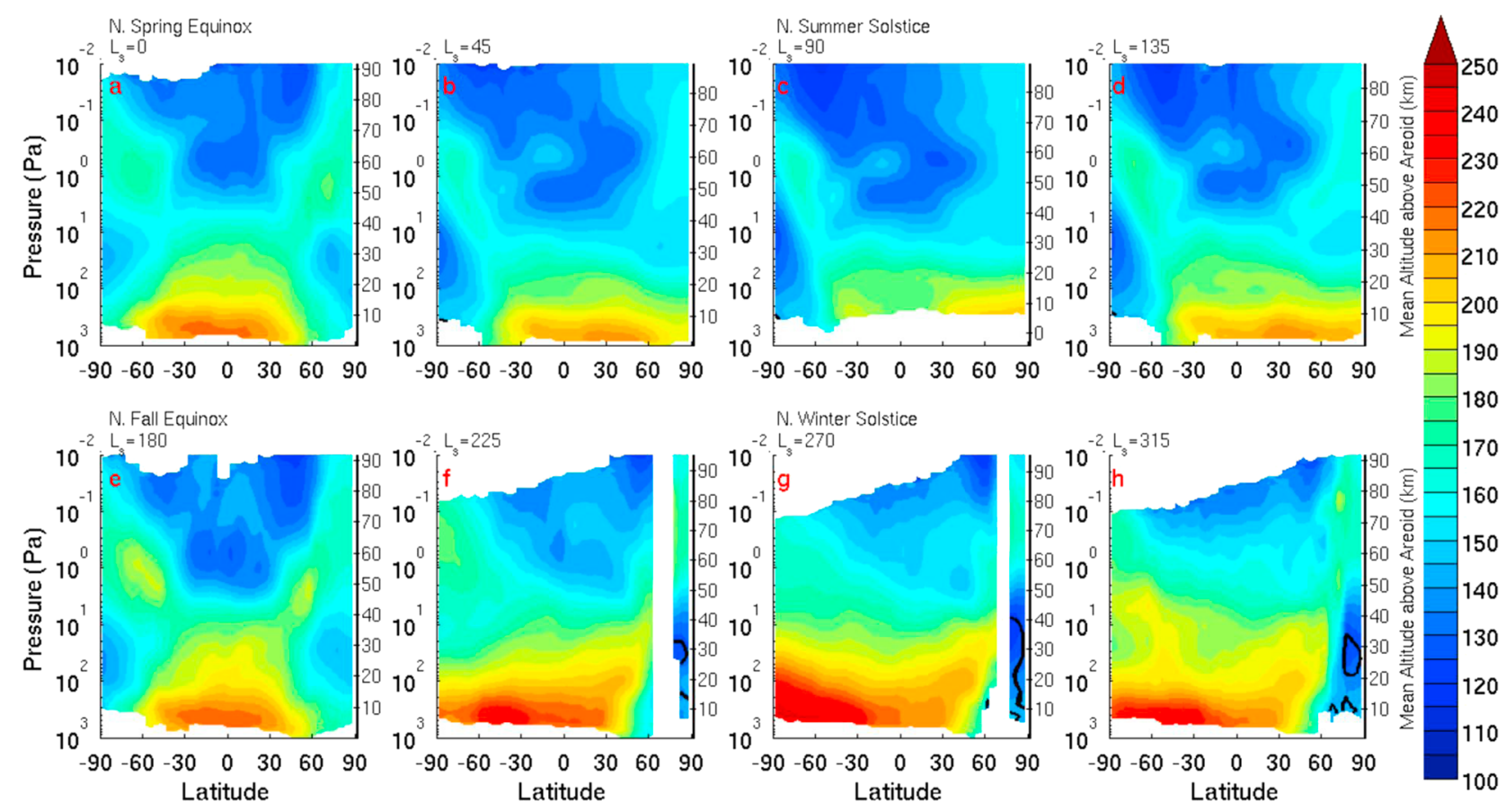

Figure 4. Zonal average temperature $(\mathrm{K})$ nightside retrievals of $M Y 29$ for the $\mathrm{L}_{\mathrm{s}}$ bins labeled at the top of each panel. Contours are every $5 \mathrm{~K}$. The black contour indicates the $\mathrm{CO}_{2}$ frost point.

[7] As described by Kleinböhl et al. [2009], the operation of the instrument while in orbit around Mars has differed somewhat from the plans described by McCleese et al. [2007] due to anomalous behavior of one of two mechanical actuators. The departure from nominal operation most important for this study occurred between 9 February 2007 and 14 June $2007\left(\mathrm{~L}_{\mathrm{s}}=180^{\circ}-257^{\circ}\right)$. In this period, MCS operated in a mode known as "limb staring" in which the limb was observed at a constant angle relative to the spacecraft, and no compensation was made for variations in the altitude of the orbit of MRO or for the figure of the planet. This degraded mode of operation affects the altitude range of the atmosphere observed by the instrument, as well as the calibration of the observed radiances. Specifically, retrievals of geophysical properties from data collected during this period provide less information about high altitudes in the southern hemisphere and both low and very high altitudes near the north pole than do retrievals from data collected at all other periods. Therefore, this study relies primarily (but not entirely) on retrievals from the 31 months of nominal operation.

[8] The percentage of longitude bins sampled in a zonal average is shown in Figure 1. The undersampling shown in Figure 1 is usually attributable one of two causes: (1) the instrument was powered off for operational reasons, or the spacecraft was pointed significantly off nadir and (2) Aerosol opacities were high, due to dust in storms and near the equator in all seasons, or due to water ice clouds in northern spring and in the summer in the northern tropics [Kleinböhl et al., 2009]. In the latter cases, sampling is continuing to improve as the data are reprocessed using increasingly sophisticated retrieval algorithms which permit retrievals under conditions of moderate aerosol opacities. Figures 2 and 3 show examples of how dayside and night- side latitude/longitude bins are populated in selected $\mathrm{L}_{\mathrm{s}}$ bins. In particular, note the differences in sampling of longitudes in adjacent latitudinal bins, especially in cases in which few longitudinal bins are sampled. This must be taken into account when evaluating zonally averaged bins.

\subsection{Winds}

[9] An estimate of the zonal gradient wind, $U(p)$, is derived from the zonal average temperature by taking the lowest pressure level with retrieved temperature data in each latitudinal bin as a level of no motion, $p_{L N M}$, and calculating the thermal wind, $\hat{U}(p)$ :

$$
\hat{U}(p)=\int_{p_{L N M}}^{p} \frac{R_{d}}{f}\left(\frac{d T}{d y}\right)_{p} d \ln p^{\prime}
$$

where $\mathrm{R}_{\mathrm{d}}$ is the specific gas constant, $f$ is the Coriolis parameter for the latitudinal bin, and $\left(\frac{d T}{d y}\right)_{p}$ is the temperature gradient at constant pressure, which is computed using interpolation. To account for the centrifugal force and thus compute the gradient wind $U(p)$, we iteratively apply equation (2) to convergence [Holton, 2004]:

$$
U_{n+1}(p)=\frac{U_{n}}{1+\frac{\sqrt{U_{n}^{2}}}{\left|f R_{M}\right|}}
$$

where $R_{M}$ is the radius of Mars. Equations (1) and (2) are only appropriate for winds in approximate geostrophic balance and so cannot be used for diagnosis of zonal winds in the tropics where the Coriolis parameter tends to zero. Therefore, $U(p)$ calculated in the tropics is not plotted. The zonal wind velocity at $p_{L N M}$ is not, in fact, zero, so the true 

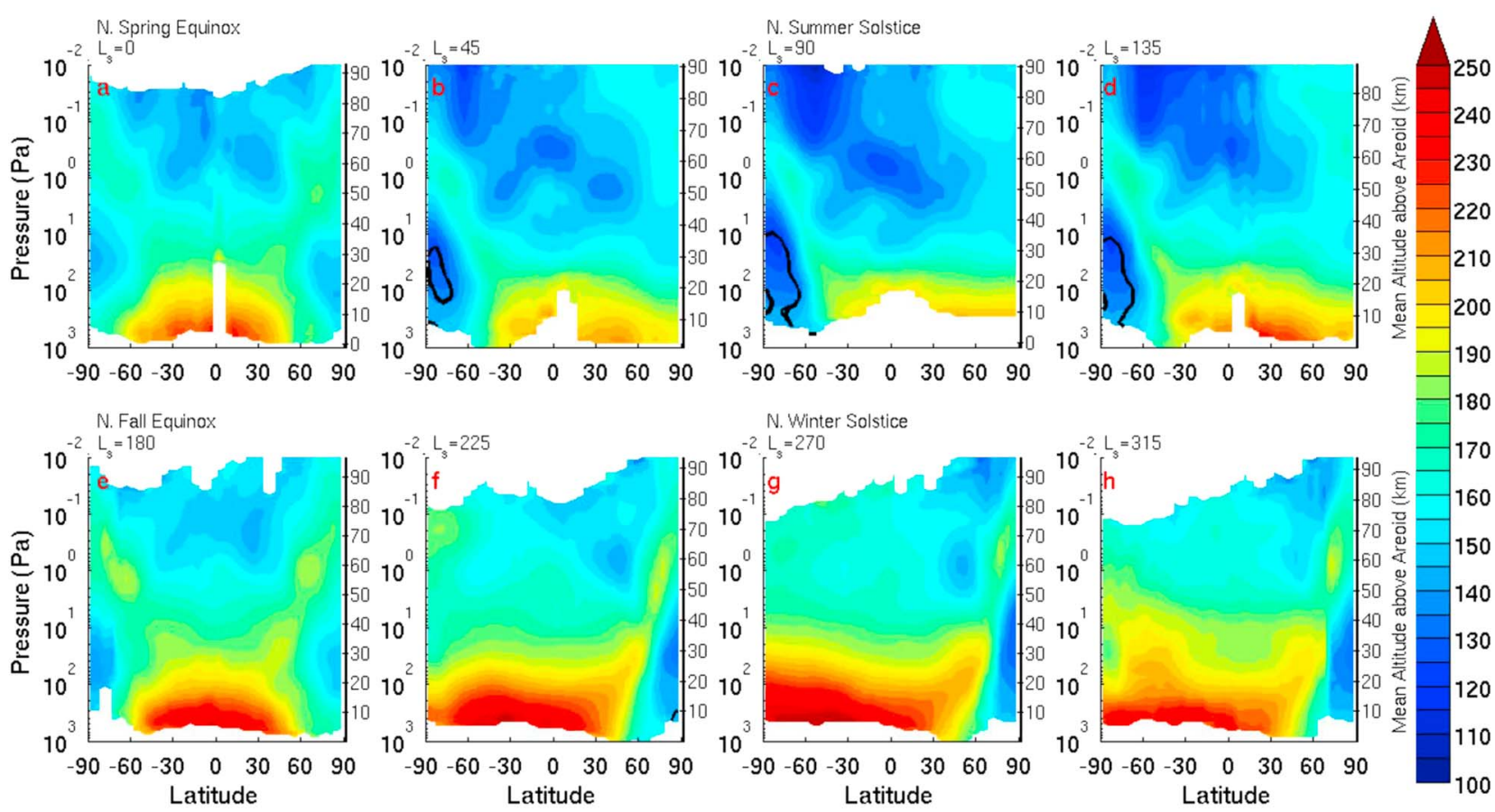

Figure 5. Zonal average temperature $(\mathrm{K})$ dayside retrievals of $M Y 29$ for the $\mathrm{L}_{\mathrm{s}}$ bins labeled at the top of each panel. Contours are every $5 \mathrm{~K}$. The black contour indicates the $\mathrm{CO}_{2}$ frost point.

zonal wind is the sum of the zonal wind velocity at $p_{L N M}$ and $U(p)$. See Smith et al. [2001] for a similar discussion.

\section{Thermal Structure and Aerosol Distribution of the Atmosphere}

\subsection{Seasonal Variability in the Thermal Structure and Implied Zonal Winds: Distinction Between the Equinoctial and Solstitial Circulations}

[10] Figures 4 and 5 show the zonal average nightside and dayside temperatures, respectively, for $\mathrm{L}_{\mathrm{s}}$ intervals corresponding to the solstices, the equinoxes, and intermediate seasons. At northern vernal equinox, Figures $4 \mathrm{a}$ and $5 \mathrm{a}$, $\left(\mathrm{L}_{\mathrm{s}}=0^{\circ}\right)$, temperatures at equatorial to subtropical latitudes generally decrease with height from near the surface to $\sim 2 \mathrm{~Pa}(\sim 55 \mathrm{~km}$ above the MOLA areoid), falling to near $140 \mathrm{~K}$ aloft. Near both poles, there are structures in the middle atmosphere $(0.1-10 \mathrm{~Pa}$, centered at $1 \mathrm{~Pa})$ which we refer to as the "polar warmings," in which temperatures are as high as $\sim 175 \mathrm{~K}$ at $\sim 1 \mathrm{~Pa}$ [French and Gierasch, 1979; Conrath et al., 2000] and. In the lower atmosphere below the polar warmings, there are pronounced temperature minima, which we refer to as the "polar vortices," though MCS observations alone cannot confirm this dynamical description. The remnant cold surface and polar vortex associated with the previous northern winter produce a hemispheric asymmetry at the time of the northern vernal equinox.

[11] Only half a season later at $\mathrm{L}_{\mathrm{s}}=45^{\circ}$ (Figures $4 \mathrm{~b}$ and $5 \mathrm{~b}$ ), the southern polar vortex has intensified, extended vertically and become nearly as cold as it will be at the winter solstice $\left(\mathrm{L}_{\mathrm{s}}=90^{\circ}\right)$ with zonal average temperatures very near or perhaps even below the deposition temperature of $\mathrm{CO}_{2}$ (indicated by the black contour). The vortex in the north is visible only as a weak temperature minimum near $10 \mathrm{~Pa}$ (near $38 \mathrm{~km}$ ). In nightside observations, a $\sim 155$ $160 \mathrm{~K}$ feature stretches from the edge of the southern polar warming to the equator at $\sim 5-0.5 \mathrm{~Pa}$. This feature, which is associated with the thermal tide [Lee et al., 2009] does not appear in dayside observations and is only seen in northern spring and summer. In Figures $4 \mathrm{c}$ and $5 \mathrm{c}$, the asymmetry of the northern summer is evident from the surface to $0.02 \mathrm{~Pa}$. The nearly symmetric thermal structure returns half a year later (Figures $4 \mathrm{e}$ and $5 \mathrm{e}$ ). The pattern of winter-summer temperatures in the atmosphere in the first half of MY 29 is clearly not repeated in the second half (Figures $4 \mathrm{f}-4 \mathrm{~h}$ and $5 \mathrm{f}-5 \mathrm{~h})$. The atmosphere is substantially warmer beginning with $\mathrm{L}_{\mathrm{s}}=225^{\circ}$.

[12] Thus, the thermal structure of Mars shown in Figures 4 and 5 varies seasonally between two modes. There is a symmetrical equinoctial structure with intense middle atmospheric polar warmings that is broad in horizontal extent overlying polar vortices of similar temperature. The solstitial structure is has a narrow winter middle atmospheric polar warming thermally connected with the lower atmosphere, a cold deep winter lower atmospheric polar vortex, and a summer middle atmospheric polar warming thermally detached from the lower atmosphere. (Our use of the terms thermal connection and detachment is purely descriptive, in which "connection" implies that an isotherm can be drawn from the lower atmosphere around the region of polar warming and "detachment" implies an isotherm of this kind cannot be constructed.) Figures 6 and 7 show the inferred zonal gradient winds corresponding to the zonal average temperatures shown in Figures 4 and 5, revealing equinoctial conditions with strong westerly jets in the extratropics of both hemispheres. Solstitial conditions are shown to be associated 

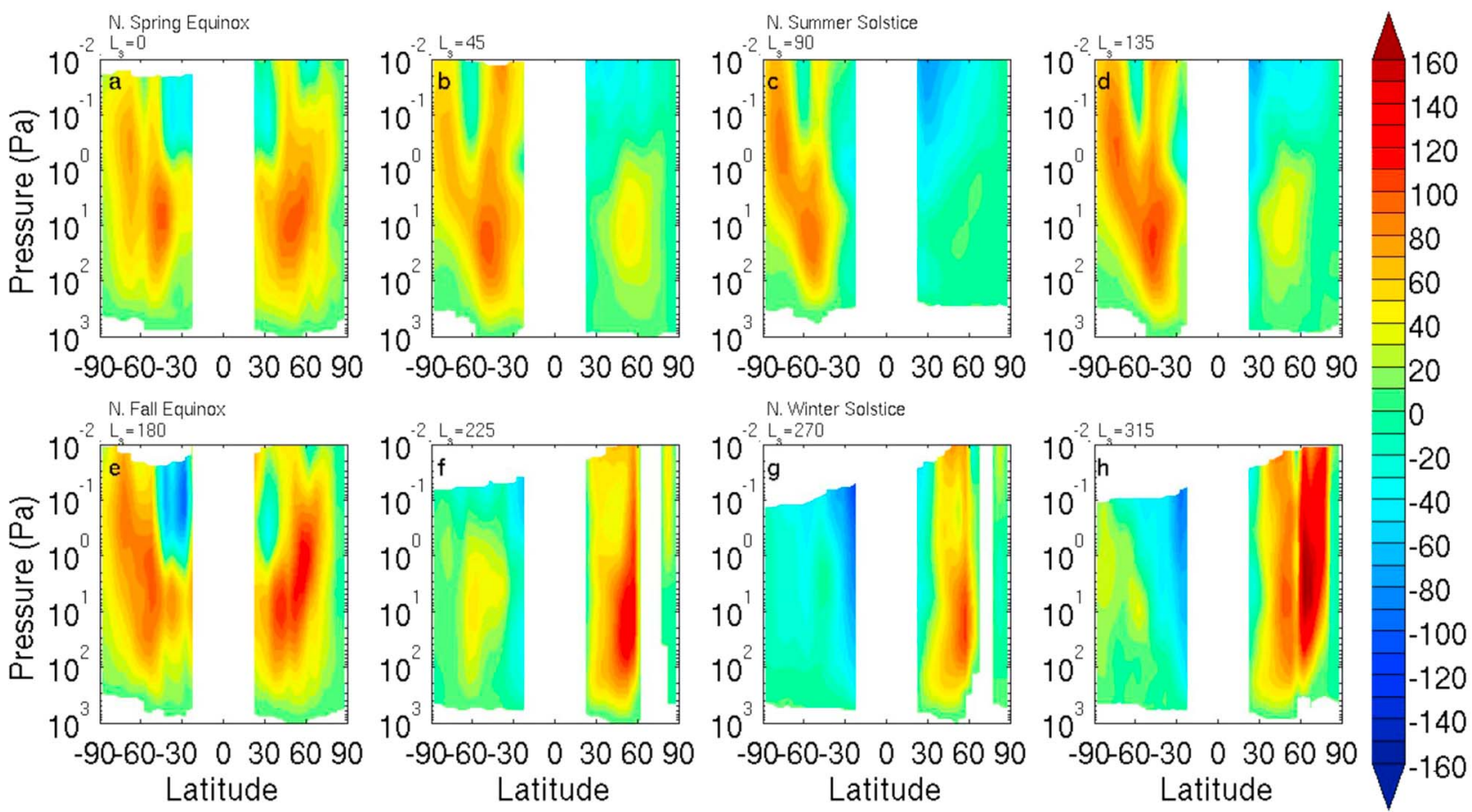

Figure 6. Estimated zonal wind velocity $\left(\mathrm{ms}^{-1}\right)$ nightside retrievals of MY 29 for the $\mathrm{L}_{\mathrm{s}}$ bins labeled at the top of each panel. Contours are shown every $10 \mathrm{~ms}^{-1}$.

with one very strong westerly jet in the extratropics of the winter hemisphere, which is stronger in northern winter than in southern winter.

[13] The seasonal variability of the intensity of westerly jets shows that the transition between the equinoctial and solstitial modes of thermal structure is gradual. The relative intensities of the westerly jets in the northern and southern hemispheres during northern spring and summer of MY 29 are shown in Figure 8a. The intensity of the southern jet begins strong and very slowly grows during this period. The
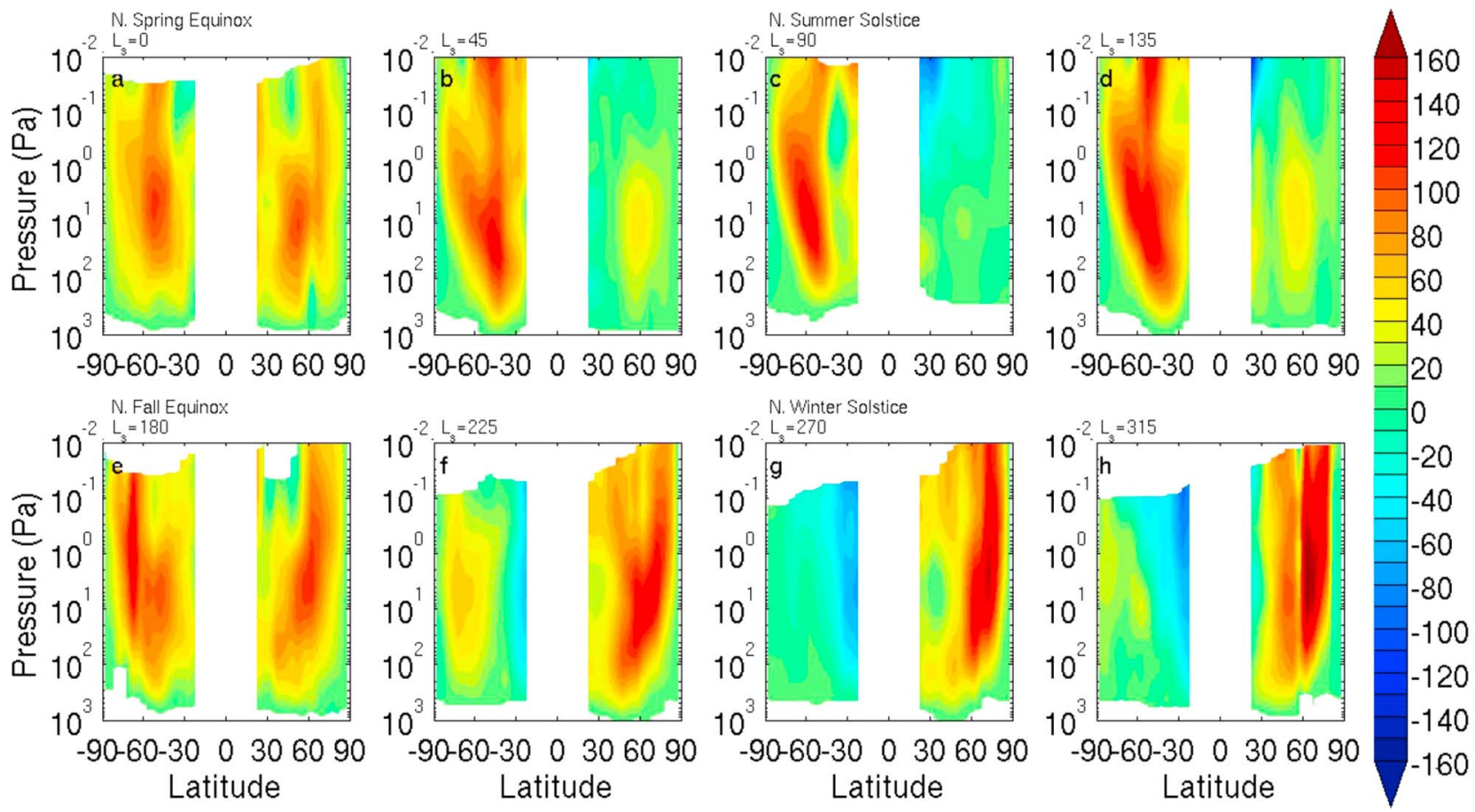

Figure 7. Estimated zonal wind velocity $\left(\mathrm{ms}^{-1}\right)$ dayside retrievals of MY 29 for the $\mathrm{L}_{\mathrm{s}}$ bins labeled at the top of each panel. Contours are shown every $10 \mathrm{~ms}^{-1}$. 

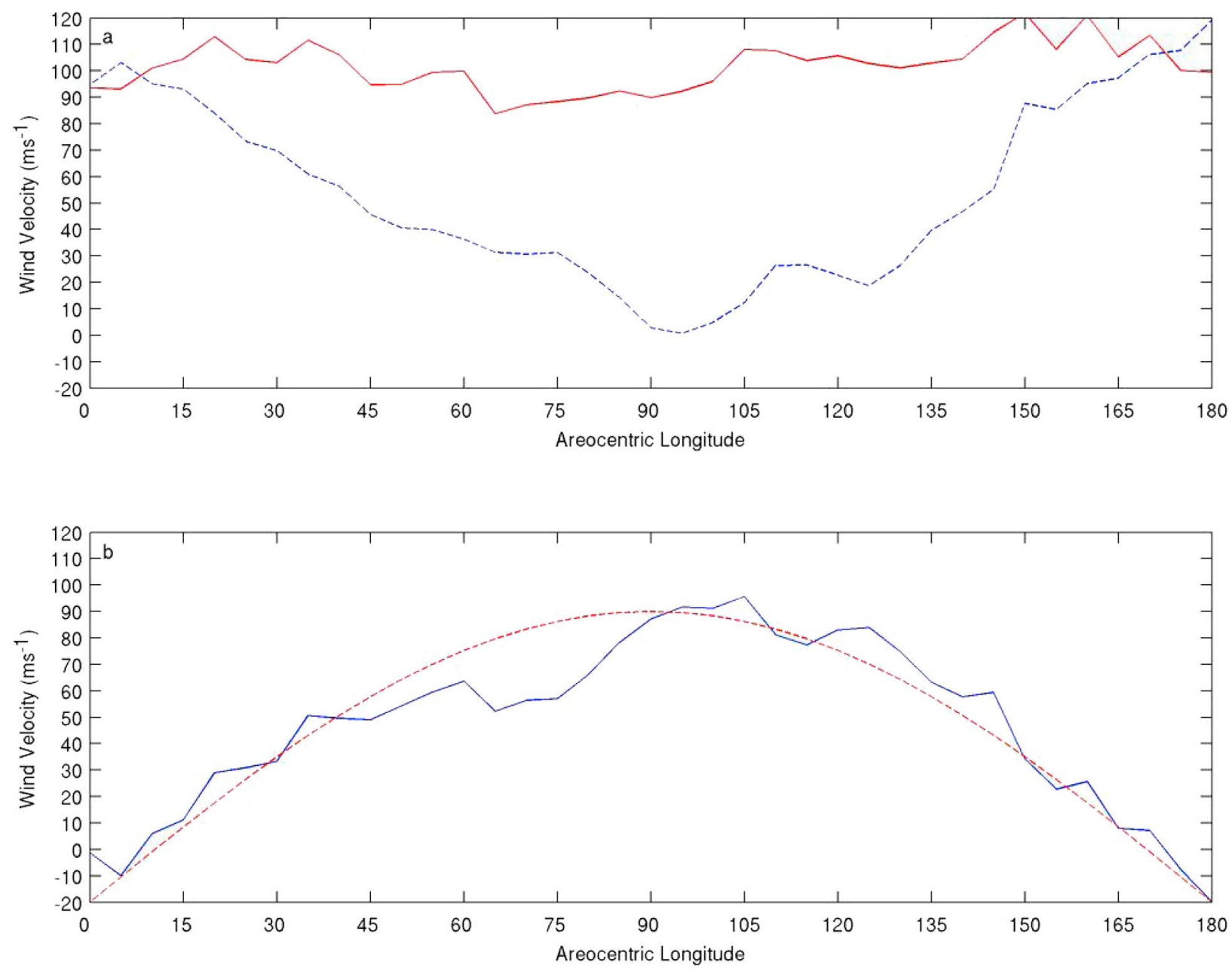

Figure 8. (a) Estimated maximum westerly jet velocity in the southern hemisphere (red solid line) and the northern hemisphere (blue dashed line) during northern spring and summer of MY 29. (b) Difference between the maximum westerly jet velocities in the southern and northern hemispheres during northern spring and summer of MY 29 (blue solid line). Red dashed line is $-20+110 \times \sin \left(\mathrm{L}_{\mathrm{s}}\right)$.

northern jet gradually decays to nothing at the northern summer solstice and then grows back to its equinoctial intensity relatively gradually except for the abrupt change around $L_{s}=140^{\circ}-145^{\circ}$. The southern jet intensity also rises abruptly during this period. Figure $8 \mathrm{~b}$ shows the difference between the southern and northern jet intensities at their respective maxima in comparison with a simple sinusoid with an extremum at northern summer solstice. As expected, the difference in jet intensity is small at the equinoxes and very large at the northern summer solstice.

[14] Figure 9 shows the variation in equatorial nightside thermal structure during northern spring and summer of MY 29. Temperatures cool from their vernal equinox values to a minimum at $\sim \mathrm{L}_{\mathrm{s}}=45^{\circ}$, remain roughly constant until after northern summer solstice, and then gradually warm until $\mathrm{L}_{\mathrm{s}}$ $=135^{\circ}$. Beginning with $\mathrm{L}_{\mathrm{s}}=135^{\circ}-145^{\circ}$, there is a more abrupt rise in temperature (observe the slope of the isotherms) of $\sim 1 \mathrm{~K} /$ degree of $\mathrm{L}_{\mathrm{s}}$ over a great depth of the middle atmosphere (2-90 Pa). Yet since the equator-pole gradient increases at roughly the same rate at both poles, this sudden warming at the equator does not affect the general sinusoidal variation in the difference in jet intensities. An abrupt change in temperatures in this latitudinal band and season was not observed in MY 28.

\subsection{Seasonal Variability of the Latitudinal Vertical Distribution of Dust}

[15] Figures 10 and 11 show the seasonal variation of the zonal average of dust density-scaled opacity (note the $\log _{10}$ dimension of the color bar). We use the density-scaled opacity (the quotient of opacity and the atmospheric density) to describe aerosol distribution because it is both proportional the mass mixing ratio and the aerosol heating rate. For reference, the zonal average dust opacity is plotted in Figure 12 (nightside) and Figure 13 (dayside). This quantity is proportional to the particle number density of dust. The latitudinal vertical distribution of dust has equinoctial and solstitial modes. The equinoctial mode is characterized by penetration of dust to high altitudes over the tropics and a lower height of penetration near the poles (Figures 10a and 12a). Similarly, the solstitial mode is characterized by penetration of dust to high altitudes over the tropics, a 


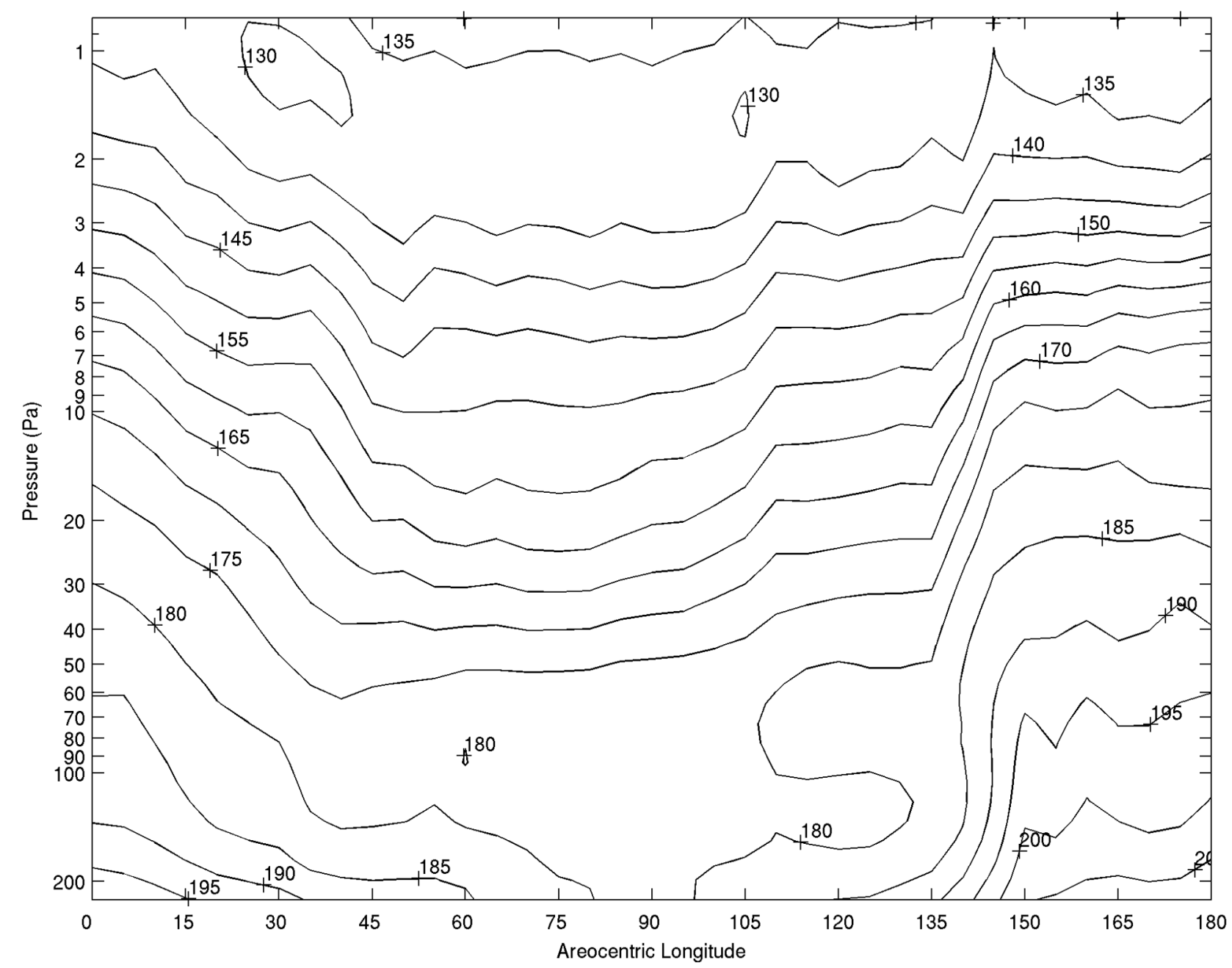

Figure 9. Zonal average nightside temperature $(\mathrm{K})$ between $5^{\circ} \mathrm{S}$ and $5^{\circ} \mathrm{N}, \mathrm{L}_{\mathrm{s}}=0^{\circ}-180^{\circ}$ for $\mathrm{MY} 29$. The pressure range is restricted for emphasis.

region of nearly dust-free air in the winter hemisphere midlatitudes which may extend all the way to the pole, and a region of moderate penetration of dust near the summer pole (Figures 10c and 12c). Although the basic morphology of the solstitial mode is similar for both solstices, quantitative aspects of particular features differ (Figures 10c and $11 \mathrm{~g})$. The clear region in the winter hemisphere extends further equatorward at northern summer solstice, while the height of the dust in the tropics and the magnitude of the dust density-scaled opacity reach their maximum at southern summer solstice. The distribution of dust during northern winter is expected to be highly variable from year to year due to the apparent chaotic nature of major dust storms, although the general trends apparent in MY 29 would be expected for most years. A similar pattern of variability in the height of dust mixing with season was observed by Anderson and Leovy [1978] and Jaquin et al. [1986] using Mariner 9 and Viking Orbiter data, respectively, though the different characteristics of the observations do not permit detailed comparison.

[16] Caution must be exercised with dust retrieved by MCS near the winter poles. The current MCS retrieval algorithm does not retrieve carbon dioxide ice [Kleinböhl et al., 2009].
Instead when $\mathrm{CO}_{2}$ ice aerosol is seen in the atmosphere, the retrieval algorithm will indicate dust. Thus the aerosols (reported as dust) in the vicinity of the winter pole when the retrieved temperatures are below the condensation temperature of $\mathrm{CO}_{2}$ may in fact be carbon dioxide ice. Note: MCS retrievals distinguish water ice from other aerosols.

[17] The zonal averages in Figures 10 and 11 are heavily biased toward the dust distributions at the longitudes where retrievals are successful. For example, the longitudinal sampling of southern spring and summer, when the atmosphere is dustier, is much less complete than in northern spring and summer. In general, zonal averages of aerosols are systematically biased toward regions/vertical ranges with low dust/ice opacities. The high air mass factor of MCS limb observations makes retrieval success unlikely at high aerosol opacities.

[18] In the tropics during northern spring and summer (Figures 10b-10d), dust density-scaled opacity, and thus the heating rate due to dust, has a maximum at pressures of 60 $120 \mathrm{~Pa}, \sim 15-25 \mathrm{~km}$. While Figures 10a-10d show gradual development of a high-altitude tropical dust maximum, its disappearance between Figures 10d and 10e appears abrupt, as can be confirmed by looking at zonal averages in the intermediate $\mathrm{L}_{\mathrm{s}}$ bins. Figure 14 shows consecutive $\mathrm{L}_{\mathrm{s}}$ bins 

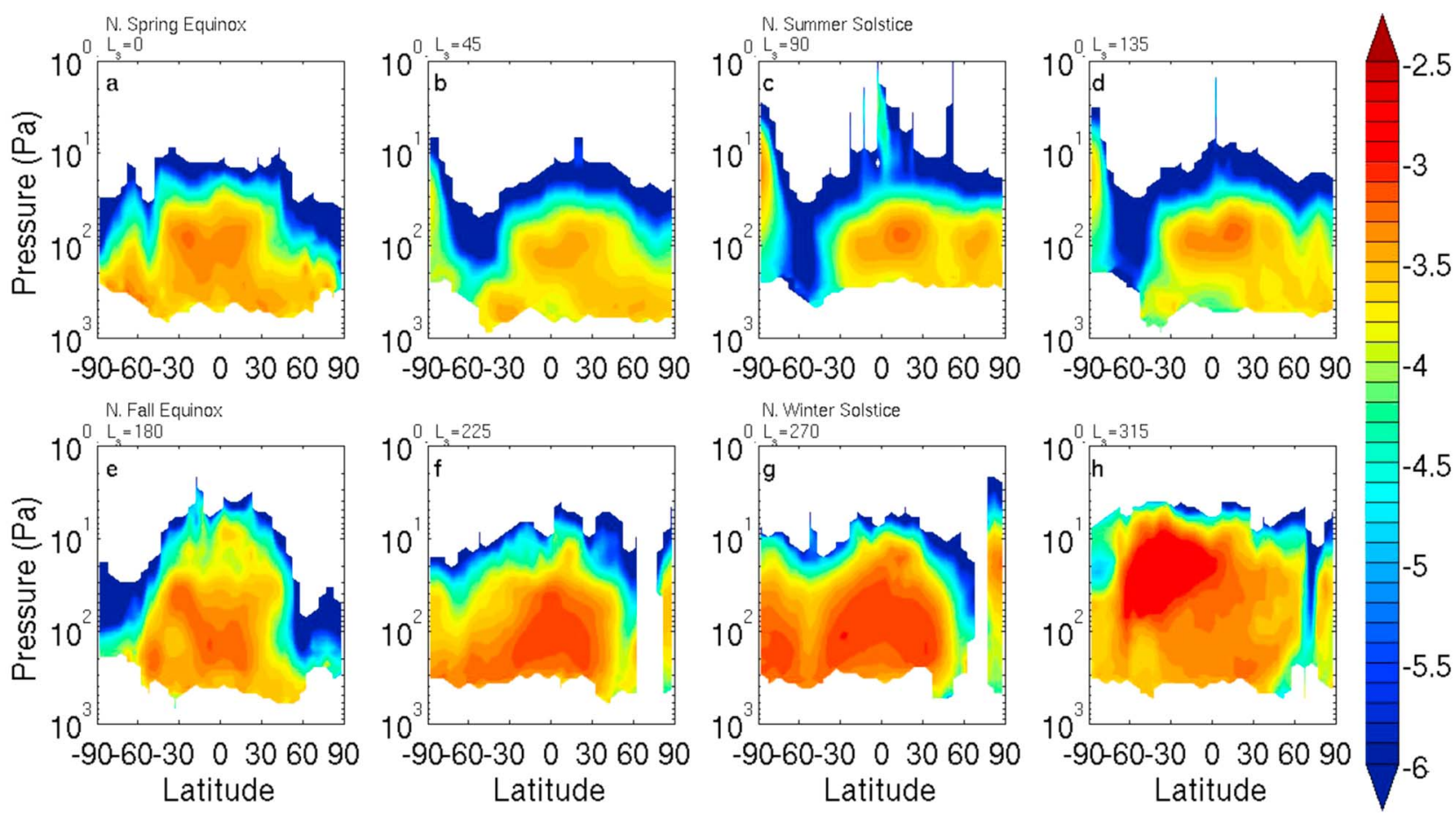

Figure 10. $\log _{10}$ of the zonal average dust density-scaled opacity $\left(\mathrm{m}^{2} \mathrm{~kg}^{-1}\right)$ nightside retrievals of MY 29 for the $L_{s}$ bins labeled at the top of each panel. Contours are shown every $0.1 \log$ units. Note the pressure scale is between 1000 and $1 \mathrm{~Pa}$.
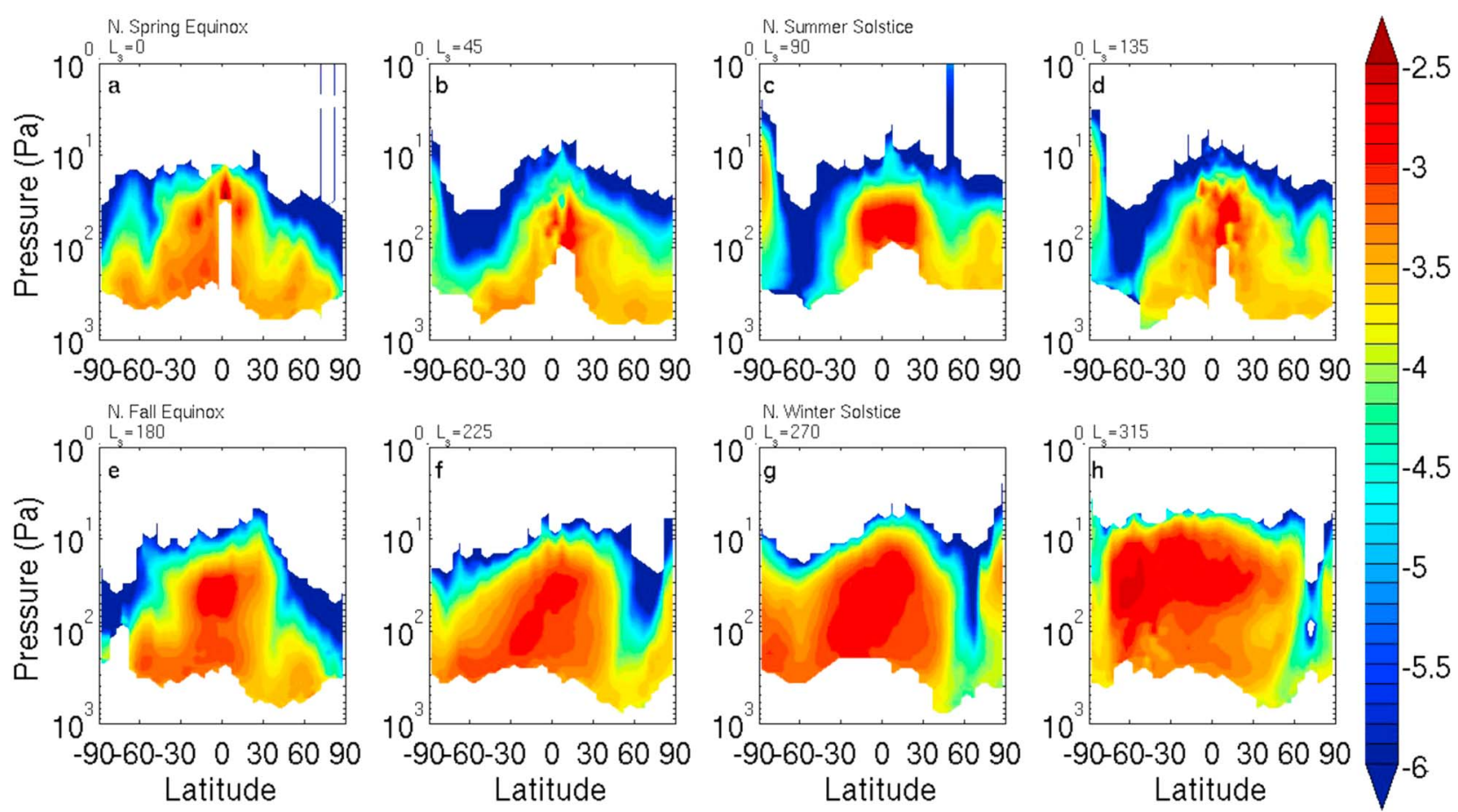

Figure 11. $\log _{10}$ of the zonal average dust density-scaled opacity $\left(\mathrm{m}^{2} \mathrm{~kg}^{-1}\right)$ dayside retrievals of MY 29 for the $L_{s}$ bins labeled at the top of each panel. Contours are shown every $0.1 \log$ units. Note the pressure scale is between 1000 and $1 \mathrm{~Pa}$. 

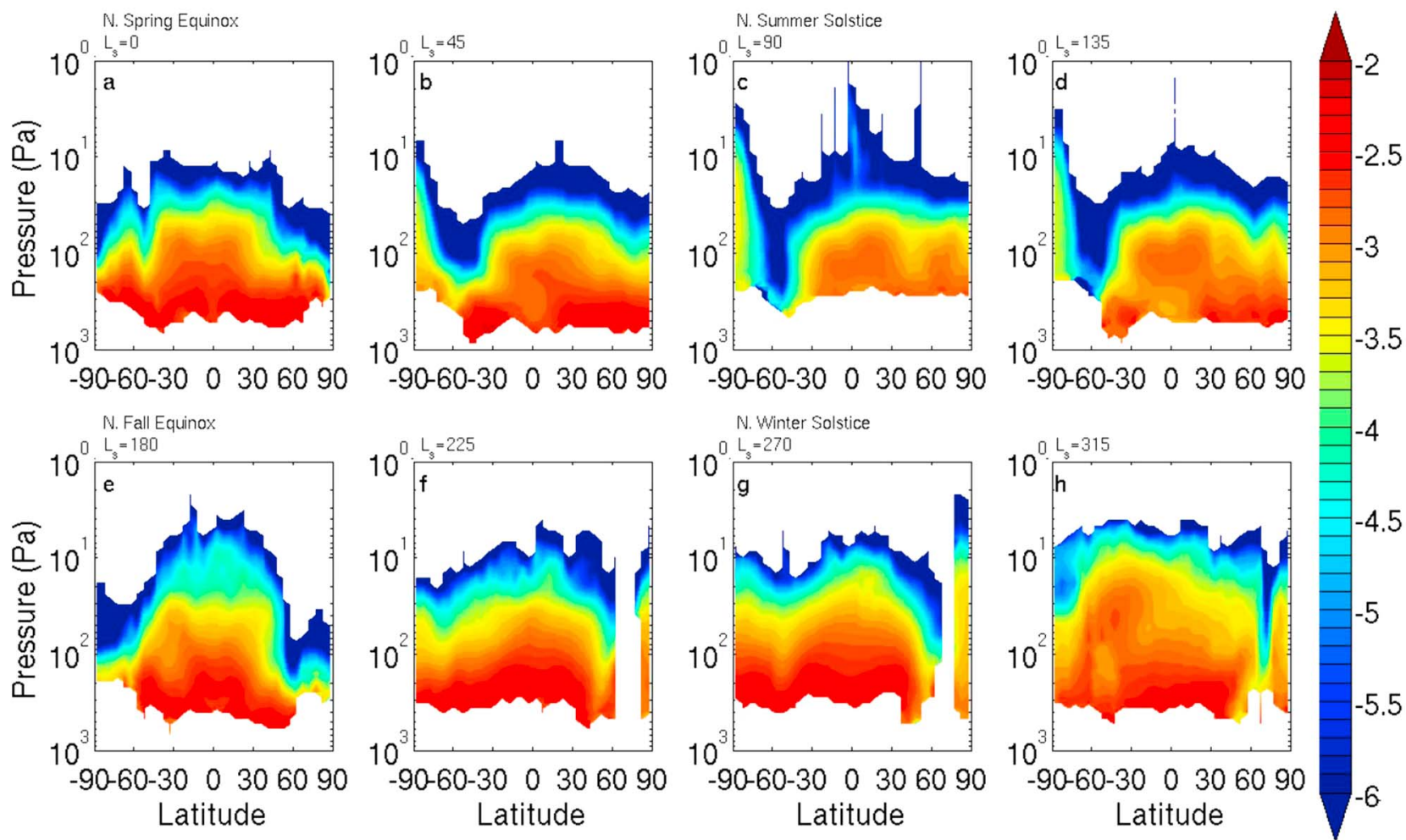

Figure 12. $\log _{10}$ of the zonal average dust opacity $\left(\mathrm{km}^{-1}\right)$ nightside retrievals of MY 29 for the $\mathrm{L}_{\mathrm{s}}$ bins labeled at the top of each panel. Contours are shown every $0.1 \mathrm{log}$ units. Note the pressure scale is between 1000 and $1 \mathrm{~Pa}$.
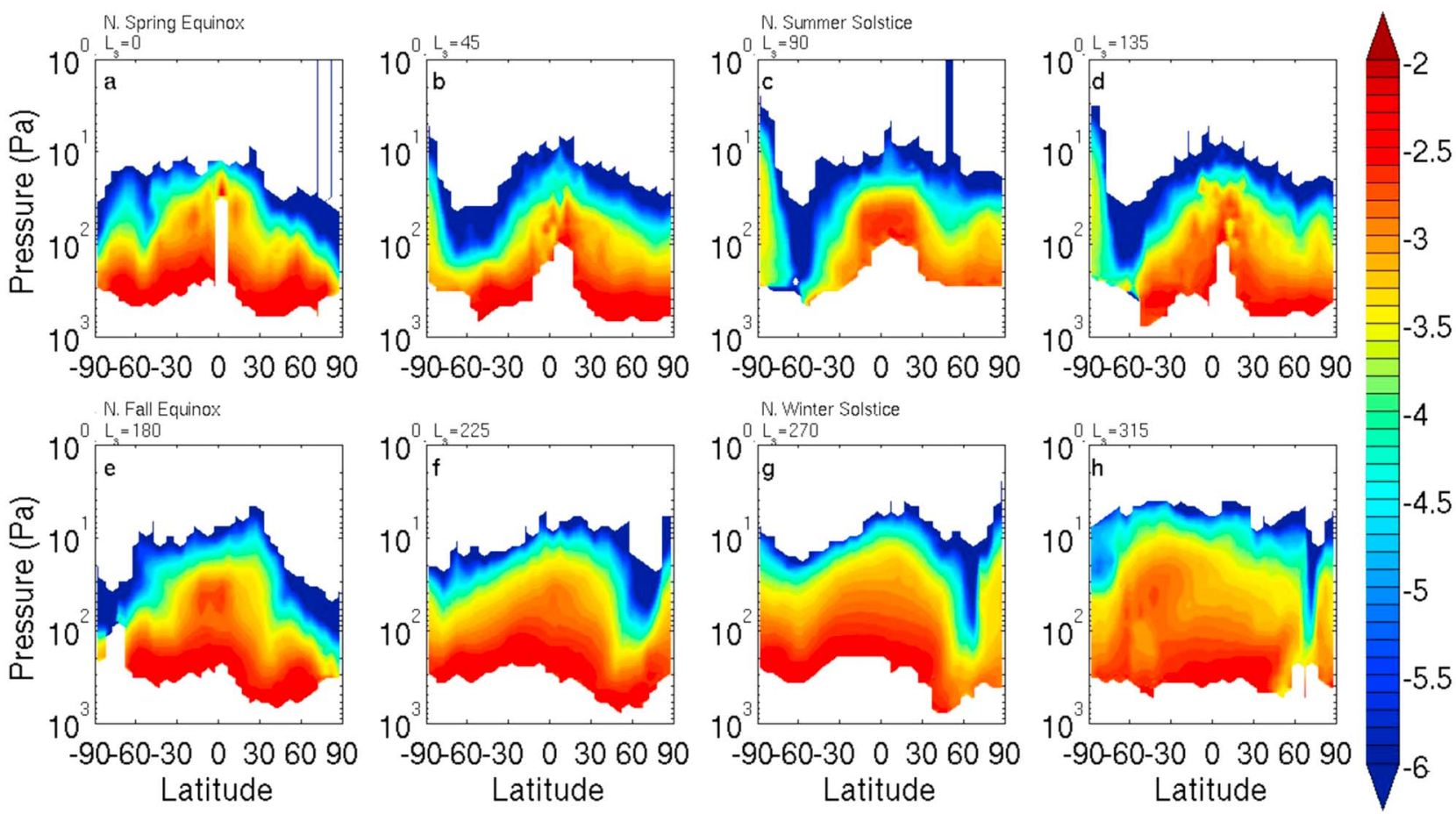

5

5

$-5.5$

Figure 13. $\log _{10}$ of the zonal average dust opacity $\left(\mathrm{km}^{-1}\right)$ dayside retrievals of MY 29 for the $\mathrm{L}_{\mathrm{s}}$ bins labeled at the top of each panel. Contours are shown every $0.1 \log$ units. Note the pressure scale is between 1000 and $1 \mathrm{~Pa}$. 

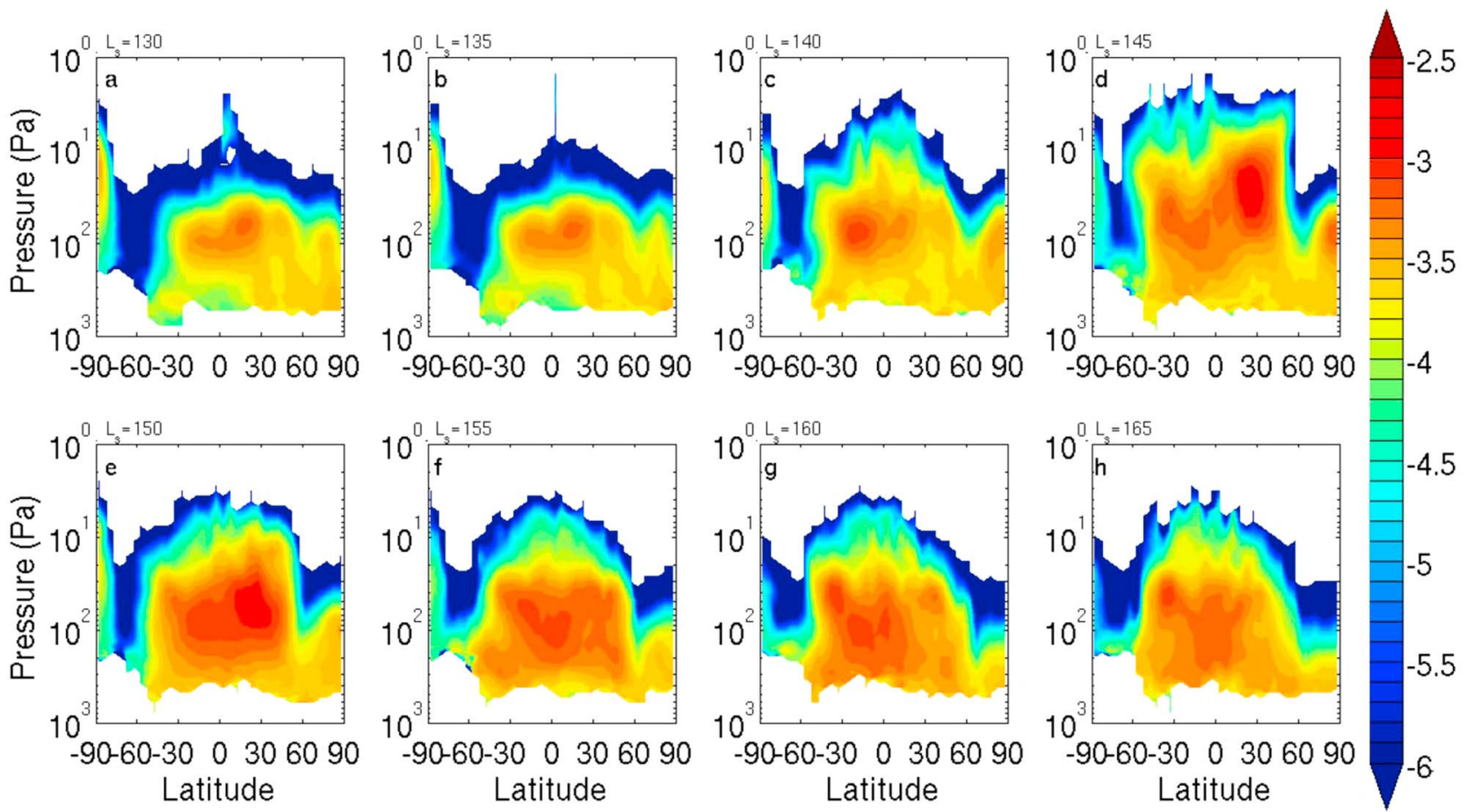

Figure 14. $\log _{10}$ of the zonal average dust density-scaled opacity $\left(\mathrm{m}^{2} \mathrm{~kg}^{-1}\right)$ nightside retrievals for the consecutive $\mathrm{L}_{\mathrm{s}}$ bins in MY 29 labeled at the top of each panel. Contours are shown every $0.1 \log$ units.

between $\mathrm{L}_{\mathrm{s}}=130$ to 165 for nightside zonal averages of dust density-scaled opacity during middle-to-late northern summer of MY 29. Between $L_{s}=130^{\circ}$ and $L_{s}=135^{\circ}$ (Figures $14 \mathrm{a}$ and $14 \mathrm{~b})$, the vertical and latitudinal distribution of dust is virtually unchanged. At $\mathrm{L}_{\mathrm{s}}=140^{\circ}$ (Figure 14c), the highaltitude tropical maximum shifts toward the southern hemisphere, but another maximum appears in the northern tropics at $\mathrm{L}_{\mathrm{s}}=145^{\circ}$ (Figure 14d) and reaches a pressure level of $\sim 30 \mathrm{~Pa}(\sim 35 \mathrm{~km})$. Figures $14 \mathrm{f}-14 \mathrm{~h}$ show a more latitudinally uniform maximum in density-scaled opacity extending from $50^{\circ} \mathrm{S}$ to $50^{\circ} \mathrm{N}$.

[19] In an effort to deconvolve the spatial and temporal variability of dust immediately following $L_{s}=130$, we show in Figure 15 cross sections of dust density-scaled opacity constructed from retrievals from five nightside MRO passes. These cross sections were selected because they contain at least one retrieval between $5^{\circ} \mathrm{S}$ and $5^{\circ} \mathrm{N}$ that crosses the equator in a narrow longitudinal band $\left(170^{\circ}-180^{\circ} \mathrm{E}\right)$ in midnorthern summer $\left(\mathrm{L}_{\mathrm{s}}=127^{\circ}-143^{\circ}\right)$. These cross sections provide snapshots over a short time interval of time of the dust distribution just west of the Martian dateline. Examining Figure 15 at the equator, there is an abrupt change in the vertical extent of the dust between $\mathrm{L}_{\mathrm{s}}=135^{\circ}$ and $141^{\circ}$, the timeframe of the change seen in Figures $10 \mathrm{~d}$ and $10 \mathrm{e}$, although there is significant variability across all of the observations comprising the cross sections. Furthermore, the individual profiles show more varied spatial structure, including very sharp vertical variations in density-scaled opacity, highlighting the importance of subscale height observations (even MCS may be smoothing these structures even at $5 \mathrm{~km}$ resolution).

[20] Figures $15 \mathrm{a}-15 \mathrm{c}$ show a strong maximum in dust mass mixing ratio at $60 \mathrm{~Pa}$ in the northern tropics. In the interval between Figures $15 \mathrm{c}$ and $15 \mathrm{~d}\left(6^{\circ}\right.$ of $\mathrm{L}_{\mathrm{s}}, \sim 12$ sols), the top of the dusty region has descended, and the dust density-scaled opacity at $60 \mathrm{~Pa}$ has fallen from near $10^{-3}$ to below $10^{-6} \mathrm{~m}^{2} \mathrm{~kg}^{-1}$. A few sols later (Figure 15e), much of the northern tropics has cleared, although a layer of dust is evident at a pressure level near $\sim 60 \mathrm{~Pa}(\sim 25 \mathrm{~km})$. From the southern midlatitudes to tropics, conditions become much dustier with time (by about 2 orders of magnitude at $100 \mathrm{~Pa}$ ) between Figures $15 \mathrm{~b}$ and $15 \mathrm{~d}$. However, dust in significant amounts is confined to north of $45^{\circ} \mathrm{S}$. Note that a large span of high southern of latitude is entirely dust free, down to at least $200 \mathrm{~Pa}$. Near the south pole, a layer of aerosol (retrieved as dust, but because it is the winter pole may be $\mathrm{CO}_{2}$ ice) emerges from the south pole extending equatorward in Figure 15b.

[21] Observations by the Mars Color Imager (MARCI) on MRO and the Thermal Emission Spectrometer (THEMIS) on Mars Odyssey suggest that the changes in dust latitudinal vertical structure depicted in Figure 14 and 15 occur at nearly the same time as the initiation of a regional dust storm in the southern tropics and subtropics; a significant increase in column optical depth is also observed in the tropics [Malin et al., 2008; Smith, 2009]. These dust events are all nearly simultaneous with the abrupt warming near the equator and the rapid increase in the intensities of the winter westerly jets discussed in section 3.1, suggesting these phenomena may be related.

[22] A high-altitude dust maximum is also present in the southern extratropics during the middle of southern summer (Figures $10 \mathrm{~g}$ and $11 \mathrm{~g}$ ) and is closer to the south pole in nightside observations. During this time, a decaying regional dust storm and associated diffuse dust haze was observed in the southern midlatitudes by MARCI [Malin 


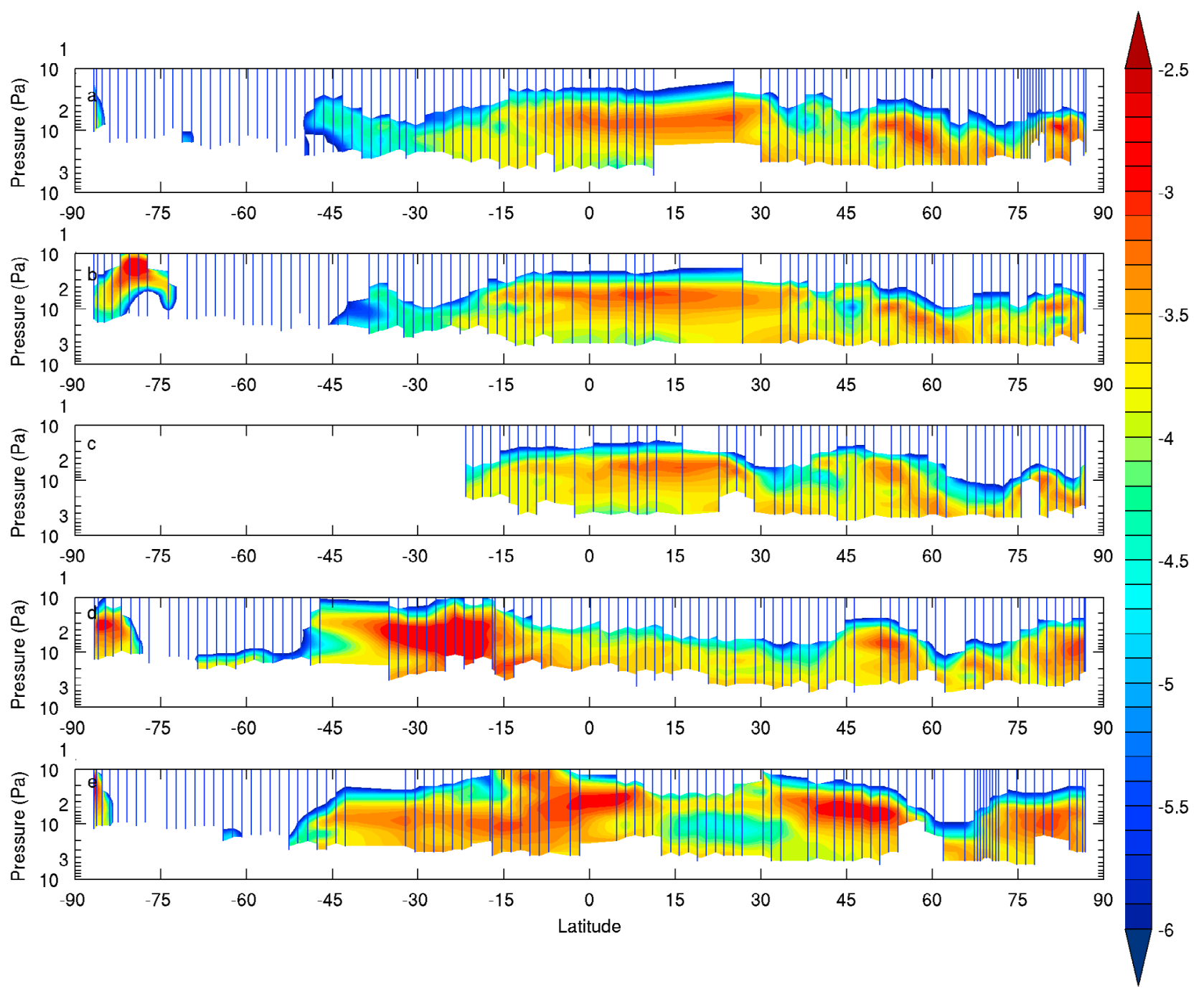

Figure 15. $\log _{10}$ of the dust density-scaled opacity $\left(\mathrm{m}^{2} \mathrm{~kg}^{-1}\right)$ in all available retrievals from five nightside MRO passes with available retrievals in a box bounded by $5^{\circ} \mathrm{S}-5^{\circ} \mathrm{N}, 170^{\circ} \mathrm{E}-180^{\circ} \mathrm{E}$ : (a) $15 \mathrm{Sep}-$ tember 2008 (orbit numbers 10024-10025), mean $\mathrm{L}_{\mathrm{s}}=127.6085^{\circ}$; (b) 21 September 2008 (orbit numbers 10103-10104), mean $\mathrm{L}_{\mathrm{s}}=130.5607^{\circ}$; (c) 2 October 2008 (orbit numbers 10235-10236), mean $\mathrm{L}_{\mathrm{s}}=135.5447^{\circ}$; (d) orbit numbers $10393-10394$, mean $\mathrm{L}_{\mathrm{s}}=141.6316^{\circ}$; (e) 14 October 2008 (orbit numbers 10446-10447), mean $\mathrm{L}_{\mathrm{s}}=143.6980^{\circ}$. The solid lines mark the mean latitude of the retrievals, and their lower end marks the highest pressure at which dust is reported. Contours are shown every 0.1 $\log$ units.

et al., 2009]. The penetration of dust over the southern summer pole is also striking; approximately $15-20 \mathrm{~km}$ higher than over the north pole during the middle of northern summer (Figures 10c-10d and 11c-11d).

\subsection{Seasonal Variability of the Latitudinal Vertical Distribution of Water Ice}

[23] Figures 16 and 17 show the seasonal variability in the zonal average of water ice density-scaled opacity on both the nightside and dayside. This quantity is proportional to the mass mixing ratio of water ice. For reference, Figures 18 and 19 show the zonal average water ice opacity. Water ice clouds are present throughout the year at various locations over the planet, and continuously over the equator. Variability between nightside and dayside averages is quite significant. Of particular note is the strong diurnal vari- ability in tropical water ice during most seasons. Wilson and Richardson [2000] argued, based on Viking Lander observations of cloud dissipation in late morning and their tidal model, that the vertical propagation of atmospheric temperature maxima and minima in the tropics is due to the diurnal tide might control cloud formation and dissipation. The basic principle is that if clouds condense at a given level of the atmosphere, that level of the atmosphere must have high relative humidity and thus is likely a local temperature minimum. Thus, as the temperature minimum propagates vertically with the tide, clouds sublimate at the original position of the temperature minimum and condense in the new position of the temperature minimum.

[24] Caution must be exercised in interpreting the zonal averages of water ice, due to the systematic bias favoring successful retrievals. In addition, ice layers at high altitudes 

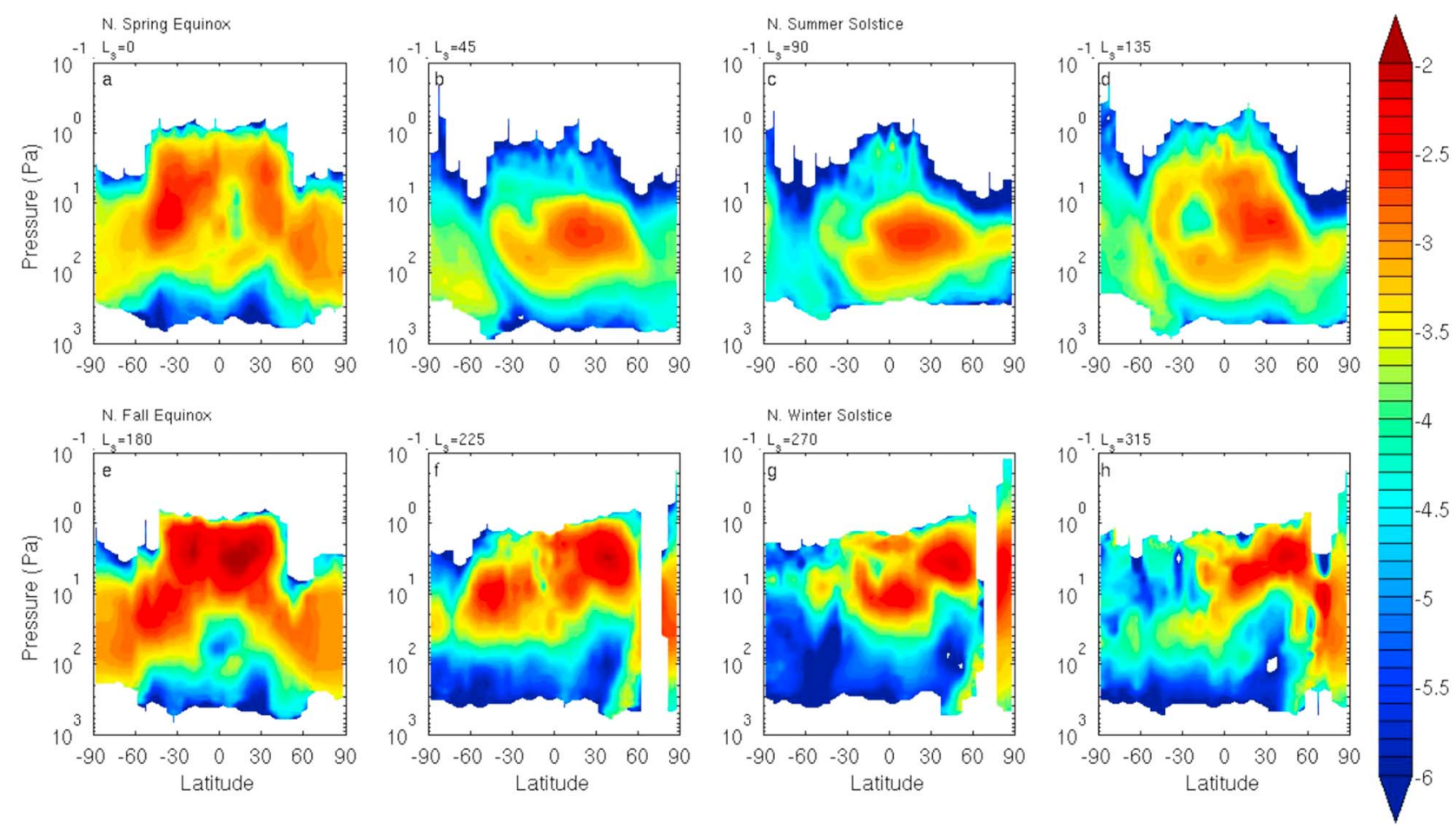

Figure 16. $\log _{10}$ of the zonal average water ice density-scaled opacity $\left(\mathrm{m}^{2} \mathrm{~kg}^{-1}\right)$ nightside retrievals of MY 29 for the $L_{s}$ bins labeled at the top of each panel. Contours are shown every $0.1 \log$ units. Note the pressure scale is between 1000 and $0.1 \mathrm{~Pa}$.
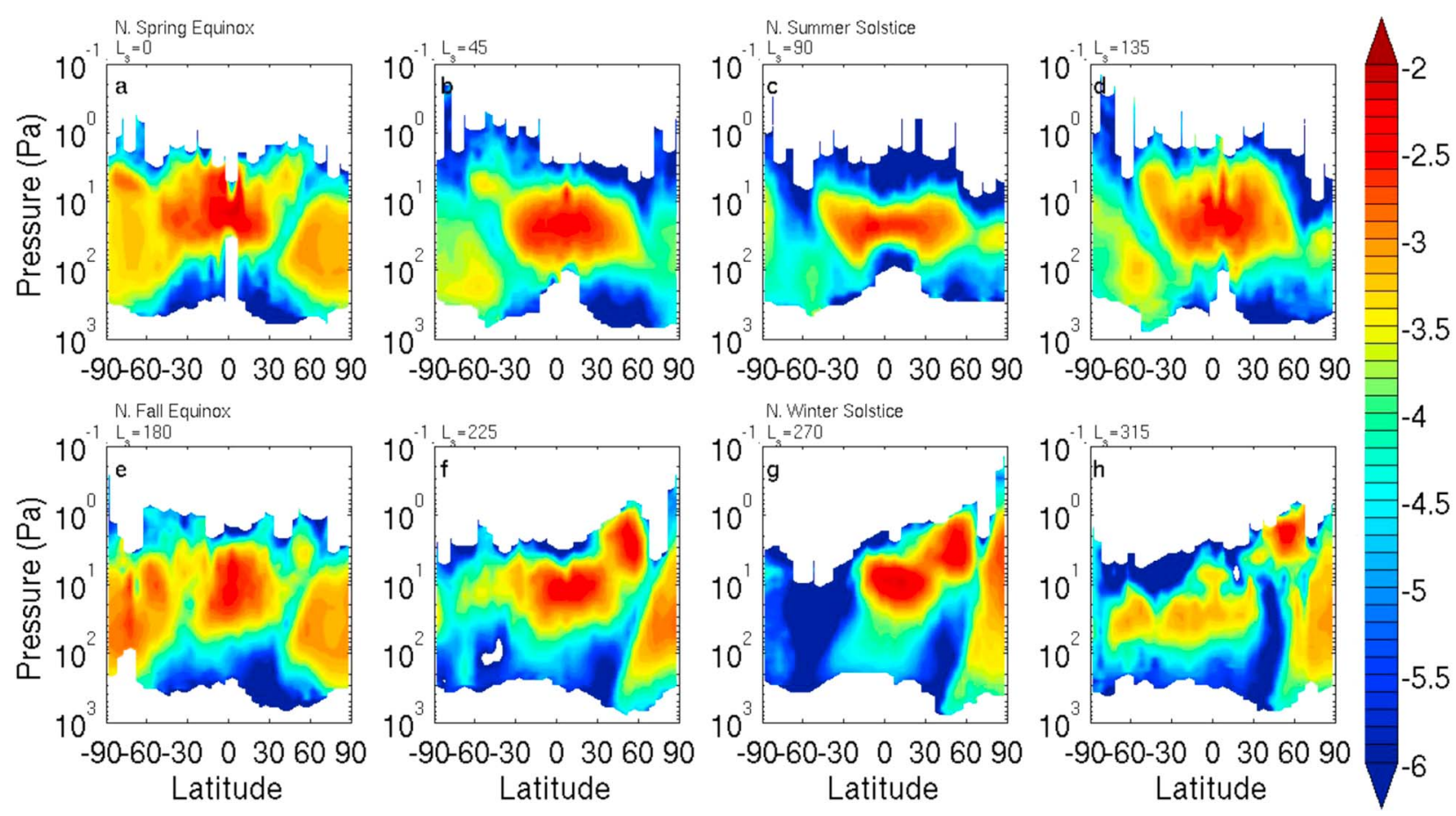

Figure 17. $\log _{10}$ of the zonal average water ice density-scaled opacity $\left(\mathrm{m}^{2} \mathrm{~kg}^{-1}\right)$ dayside retrievals for MY 29 for the $\mathrm{L}_{\mathrm{s}}$ bins labeled at the top of each panel. Contours shown are every 0.1 log units. Note the pressure scale is between 1000 and $0.1 \mathrm{~Pa}$. 

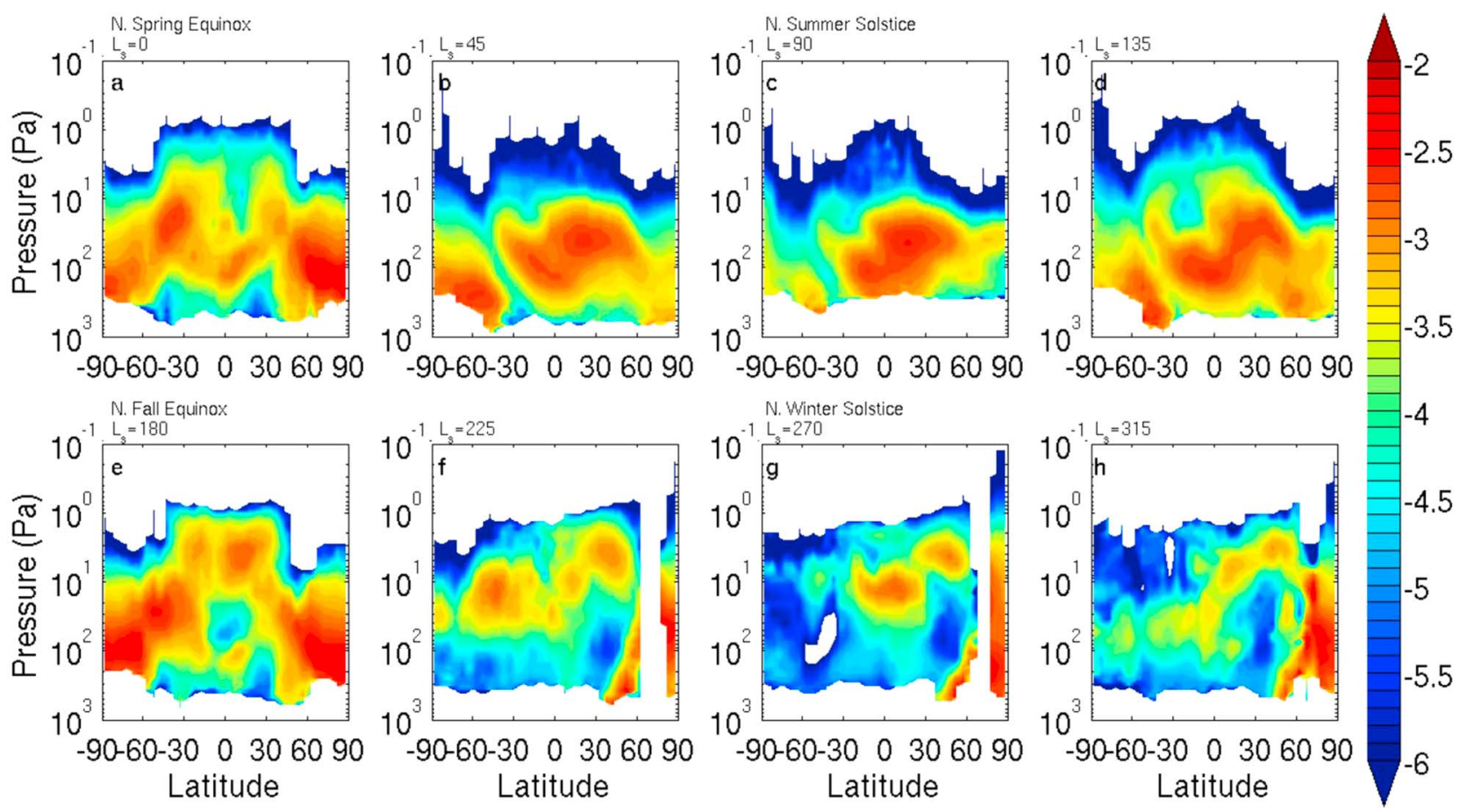

Figure 18. $\log _{10}$ of the zonal average water ice opacity $\left(\mathrm{km}^{-1}\right)$ nightside retrievals of MY 29 for the $\mathrm{L}_{\mathrm{s}}$ bins labeled at the top of each panel. Contours are shown every $0.1 \log$ units. Note the pressure scale is between 1000 and $0.1 \mathrm{~Pa}$.
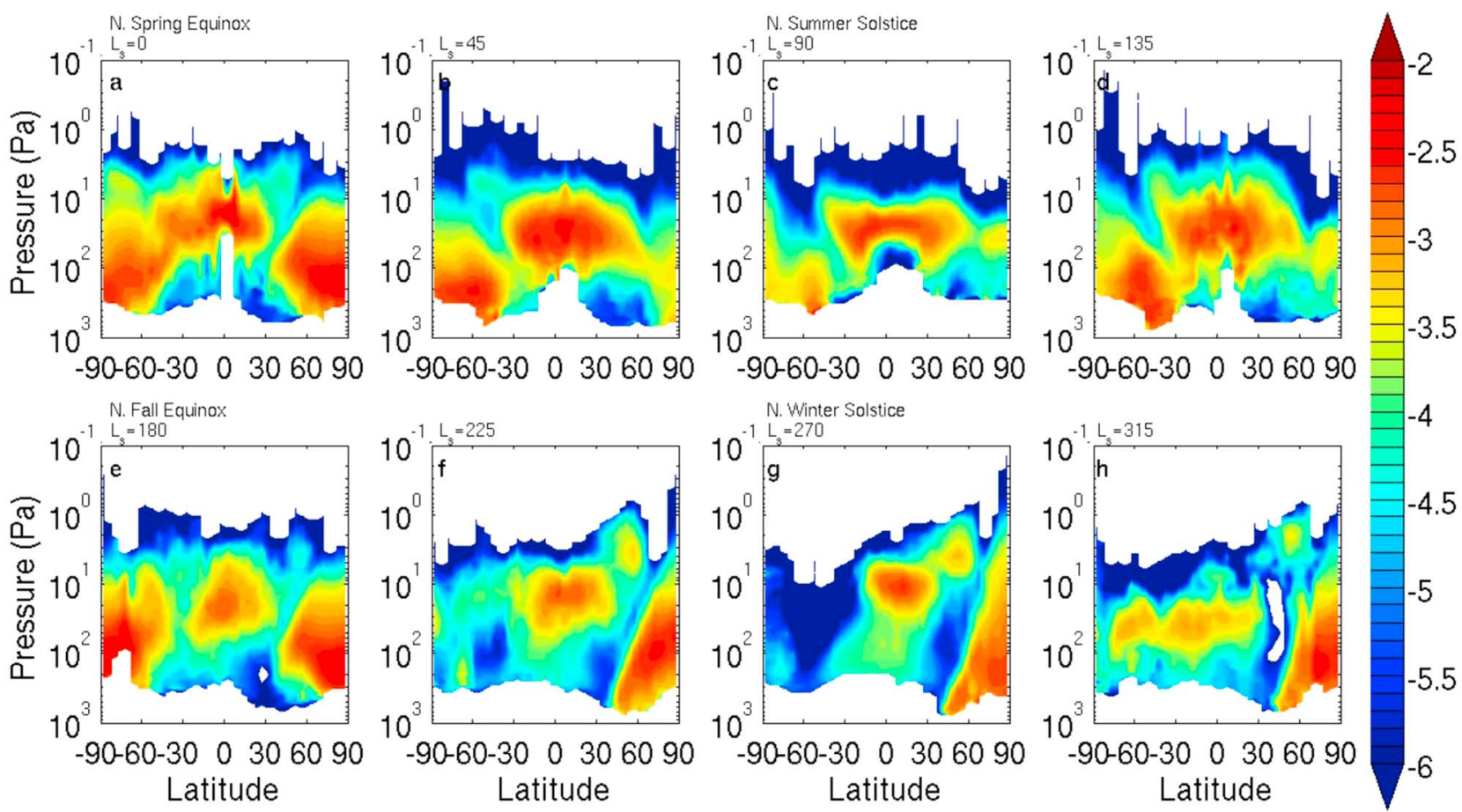

Figure 19. $\log _{10}$ of the zonal average water ice opacity $\left(\mathrm{km}^{-1}\right)$ dayside retrievals of MY 29 for the $\mathrm{L}_{\mathrm{s}}$ bins labeled at the top of each panel. Contours are shown every $0.1 \log$ units. Note the pressure scale is between 1000 and $0.1 \mathrm{~Pa}$. 
may be missed, especially when there is a layer of ice greatly detached from a more significant layer lower in the atmosphere, since the top level at which water ice is retrieved is set by a radiance-based criterion [Kleinböhl et al., 2009].

[25] At northern spring equinox, water ice on the nightside is distributed from $60^{\circ} \mathrm{S}$ to $90^{\circ} \mathrm{N}$ (Figure 16a) and extending from the bottom of MCS's observable range to a pressure level near $1 \mathrm{~Pa}(\sim 60 \mathrm{~km})$. Multiple broad maxima in the density-scaled opacity is seen over these latitudes and pressures, with the largest values occurring at $15 \mathrm{~Pa}$ and $40^{\circ} \mathrm{S}$. On the dayside (Figure 17a), water ice is more tightly confined in latitude and extending over a narrower range of pressure. In addition, a well defined minimum in water ice now separates the tropical water ice from the northern polar water ice. This minimum tilts from $30^{\circ} \mathrm{N}$ at $100 \mathrm{~Pa}$ to $50^{\circ} \mathrm{N}$ at $5 \mathrm{~Pa}$. A similar feature appears both in the north and south at many seasons, especially on the dayside.

[26] Half a season later (Figures 16b and 17b), significantly less water ice is seen at high latitudes, and the highest density-scaled opacities on both the nightside and dayside are confined and centered at the equator. At northern summer solstice (Figures $16 \mathrm{c}$ and $17 \mathrm{c}$ ), the basic pattern of $\mathrm{L}_{\mathrm{s}}=45^{\circ}$ continues, though there is a clearing of ice at $60 \mathrm{~Pa}$ over the equator on the dayside. Again, a water ice minimum tilting toward the pole with lower pressure separates the tropical water ice from the polar water ice in dayside retrievals. At this season, past observations suggest the subtropical cloud belt (called the "aphelion cloud belt" by some authors) reaches its zenith soon after northern summer solstice.

[27] A half season later (Figures $16 \mathrm{~d}$ and 17d), the structure of the subtropical cloud belt has changed. The top of the ice clouds in the tropics is substantially higher. Water ice has increased at both poles, though the ice in the southern polar region is separated from the ice in the tropics by a water ice minimum on the dayside. The increase in nightside water ice below $100 \mathrm{~Pa}$ between $\mathrm{L}_{\mathrm{s}}=90^{\circ}-135^{\circ}$ observed by MCS at northern polar latitudes is consistent with observations of a considerable increase in water ice clouds by the Mars Phoenix Lander at its landing site near $70^{\circ} \mathrm{N}$ after $\sim \mathrm{L}_{\mathrm{s}}=111^{\circ}$ [Whiteway et al., 2009].

[28] The distribution of ice at the northern autumnal equinox (Figures 16e and 17e) is similar to the distribution of ice at the northern vernal equinox (Figures 16a and 17a), except the concentrations of water ice above $10 \mathrm{~Pa}$ on the nightside are significantly greater. One explanation for this asymmetry between the equinoctial distributions is that there is no seasonal carbon dioxide ice cap over the north pole during summer, exposing the water ice cap and enhancing water vapor flux to the atmosphere in this season [Jakosky, 1985].

[29] This asymmetry between north and south continues throughout the remainder of the year (Figures $16 \mathrm{f}-16 \mathrm{~h}$ and 17f-17h). Water ice density-scaled opacities are higher in this period. The atmosphere at pressures higher than $80 \mathrm{~Pa}$ in nightside observations is generally ice free from the southern pole to $60^{\circ} \mathrm{N}$ at $\mathrm{L}_{\mathrm{s}}=225^{\circ}$ through $\mathrm{L}_{\mathrm{s}}=315^{\circ}$.

\section{Summary}

[30] We have described the seasonal variations of the zonal mean atmospheric fields, spanning more than a Martian year, providing the first systematic mapping of the lower and middle atmosphere. This is the minimum extent of the atmosphere necessary to properly represent the dynamics of the lower and middle atmosphere in general circulation models [Wilson, 1997; Forget et al., 1999].

[31] The thermal structure of the atmosphere is shown in MCS observations to vary seasonally between two modes: a symmetrical equinoctial structure with intense atmospheric polar warming overlying polar vortices; and a solstitial structure with a middle atmospheric warming over the winter pole, and a cold deep winter lower atmospheric polar vortex.

[32] MCS has provided global and seasonal vertical profiles of aerosols (dust and water ice) at the same vertical resolution $(\sim 5 \mathrm{~km})$ as the retrieved temperature profiles. These profiles reveal unanticipated characteristics in the aerosol distributions. For dust, the observations show a vertical distribution during some seasons that significantly deviates from an idealized profile constructed from the balance between sedimentation and vertical eddy diffusion [Conrath, 1975]. While the lowest reaches of the atmosphere are dustier than those of the middle atmosphere, the vertical dust distribution during most of northern spring and summer appears to contain a maximum in dust mass mixing ratio away from the surface. At roughly $15-25 \mathrm{~km}$ above the surface, above the maximum boundary layer depth, the existence of this maximum suggests that current understanding of the mechanisms by which dust enters and leaves the atmosphere is incomplete.

[33] Dust profiles from MCS also reveal in unprecedented detail considerable complexity and significant temporal and spatial variability of the dust distribution. Multiple layers of dust can be seen in cross sections of MCS data. The dust profiles also show that the atmosphere can be nearly dust free in certain regions and seasons.

[34] MCS observations yield the first vertically resolved, seasonal mapping of the distribution of atmospheric water ice. The retrieved profiles show water ice cross sections whose vertical and latitudinal distribution varies significantly with both the seasonal and diurnal cycles. The diurnal cycle variation is largely a product of the temperature variations associated with the strong diurnal tide. Indeed, Lee et al. [2009] and Benson et al. [2010] have used MCS data to show that the diurnal variation in ice opacity distribution correspond with diurnal temperature variations, which in turn show a pattern very close to that predicted for the vertically propagating tide. Seasonal variations reflect the variation of both water vapor and thermal structure. In general, the tropical water ice cloud is higher and the density-scaled opacity greater in southern summer than in northern summer. The higher cloud in the southern summer atmosphere is associated with the much warmer temperatures, and the cloud base and top are generally separated by a roughly order of magnitude in pressure, yielding an extended vertical haze. Near the solstices, the cloud belt extends horizontally throughout the tropics and into the lower midlatitudes. A well-defined break or clearing of water ice opacity occurs between mid latitudes and the winter pole.

[35] Acknowledgments. We would like to thank Tina Pavlicek for her contributions to MCS instrument operations and Mark Apolinski for his work on processing the MCS data. We also wish to thank Wayne Hartford and Mark Foote for their contributions to the design and fabrication of the instrument and the MRO spacecraft operations teams who make 
this investigation possible. Work at the Jet Propulsion Laboratory, California Institute of Technology, was performed under a contract with NASA.

\section{References}

Anderson, E., and C. Leovy (1978), Mariner 9 television limb observations of dust and ice hazes on Mars, J. Atmos. Sci., 35(4), 723-734.

Benson, J. L., D. M. Kass, A. Kleinböhl, D. J. McCleese, J. T. Schofield, and F. W. Taylor (2010), Mars' south polar hood as observed by the Mars Climate Sounder, J. Geophys. Res., doi:10.1029/2009JE003554, in press.

Clancy, R. T., B. J. Sandor, M. J. Wolff, P. R. Christensen, M. D. Smith, J. C. Pearl, B. J. Conrath, and R. J. Wilson (2000), An intercomparison of ground-based millimeter, MGS TES, and Viking atmospheric temperature measurements: Seasonal and interannual variability of temperatures and dust loading in the global Mars atmosphere, J. Geophys. Res., 105(E4), 9553-9571.

Conrath, B. J. (1975), Thermal structure of the Martian atmosphere during the dissipation of the dust storm of 1971, Icarus, 24, 36-46.

Conrath, B. J., J. C. Pearl, M. D. Smith, W. C. Maguire, P. R. Christensen, S. Dason, and M. S. Kaelberer (2000), Mars Global Surveyor Thermal Emission Spectrometer (TES) observations: Atmospheric temperatures during aerobraking and science phasing, J. Geophys. Res., 105(E4), 9509-9519, doi:10.1029/1999JE001095.

Forget, F., F. Hourdin, R. Fournier, C. Hourdin, O. Talagrand, M. Collins, S. R. Lewis, P. L. Read, and J.-P. Huot (1999), Improved general circulation models of the Martian atmosphere from the surface to above 80 km, J. Geophys. Res., 104, 24,155-24,175.

Forget, F., F. Montmessin, J.-L. Bertaux, F. Gonzalez-Galindo, S. Lebonnois, E. Quemerais, A. Reberac, E. Dimarellis, and M. A. Lopez Valverde (2009), The density and temperatures of the upper Martian atmosphere measured by stellar occultations with Mars Express SPICAM, J. Geophys. Res., 114, E01004, doi:10.1029/2008JE003086.

French, R. G., and P. J. Gierasch (1979), The Martian polar vortex: Theory of seasonal variation and observations of Eolian features, J. Geophys. Res., 84(B9), 4634-4642, doi:10.1029/JB084iB09p04634.

Hinson, D. P., M. D. Smith, and B. J. Conrath (2004), Comparison of atmospheric temperatures obtained through infrared sounding and radio occultation by Mars Global Surveyor, J. Geophys. Res., 109, E12002, doi:10.1029/2004JE002344.

Holton, J. R. (2004), An Introduction to Dynamic Meteorology, 4th ed., 535 pp., Elsevier, Amsterdam.

Jakosky, B. M. (1985), The seasonal cycle of water on Mars, Space Sci. Rev., 41(1-2), 131-200, doi:10.1007/BF00241348.

Jaquin, F., P. Gierasch, and R. Kahn (1986), The vertical structure of limb hazes in the Martian atmosphere, Icarus, 72, 528-534.

Keating, G. M., et al. (1998) The structure of the upper atmosphere of Mars: In situ accelerometer measurements from Mars Global Surveyor Science, 13, 1672-1676, doi:10.1126/science.

Kleinböhl, A., et al. (2009), Mars Climate Sounder limb profile retrieval of atmospheric temperature, pressure, dust, and water ice opacity, J. Geophys. Res., 114, E10006, doi:10.1029/2009JE003358.

Lee, C., et al. (2009), Thermal tides in the Martian middle atmosphere as seen by the Mars Climate Sounder, J. Geophys. Res., 114, E03005, doi:10.1029/2008JE003285.
Malin, M. C., B. A. Cantor, D. E. Shean, M. R. Kennedy, and T. N. Harrison (2008), MRO MARCI weather report for the week of 3 November 2008-9 November 2008, Captioned Image Release, MSSS-58, Malin Space Sci. Syst., San Diego, Calif. (Available at http://www.msss.com/msss_images/2008/11/12/.)

Malin, M. C., B. A. Cantor, M. R. Kennedy, D. E. Shean, and T. N. Harrison (2009), MRO MARCI weather report for the week of 27 July 2009-2 August 2009, Captioned Image Release, MSSS-94, Malin Space Sci. Syst., San Diego, Calif. (Available at http://www.msss.com/msss images/2009/08/05/.)

McCleese, D. J., J. T. Schofield, F. W. Taylor, S. B. Calcutt, M. C. Foote, D. M. Kass, C. B. Leovy, D. A. Paige, P. L. Read, and R. W. Zurek (2007), Mars Climate Sounder: An investigation of thermal and water vapor structure, dust and condensate distributions in the atmosphere, and energy balance of the polar regions, J. Geophys. Res., 112, E05S06, doi:10.1029/2006JE002790.

McCleese, D. J., et al. (2008), Intense polar temperature inversion in the middle atmosphere on Mars, Nat. Geosci., 1, 745-749, doi:10.1038/ ngeo332.

Smith, M. D. (2004), Interannual variability in TES atmospheric observations of Mars during 1999-2003, Icarus, 167, 148-165.

Smith, M. D. (2009), THEMIS observations of Mars aerosol optical depth from 2002-2008, Icarus, 202(2), 444-452, doi:10.1016/j.icarus.2009. 03.027 .

Smith, M. D., J. C. Pearl, B. J. Conrath, and P. R. Christensen (2001), Thermal Emission Spectrometer results: Mars atmospheric thermal structure and aerosol distribution, J. Geophys. Res., 106, 23,929-23,945.

Whiteway, J. A., et al. (2009), Mars water-ice clouds and precipitation, Science, 325(5936), 68-70, doi:10.1126/science.1172344.

Wilson, R. J. (1997), A general circulation model simulation of the Martian polar warming, Geophys. Res. Lett., 24(2), 123-126.

Wilson, R. J., and M. I. Richardson (2000), The Martian atmosphere during the Viking mission, I: Infrared measurements of atmospheric temperatures revisited, Icarus, 145 , 555-579.

Zurek, R. W., and S. E. Smrekar (2007), An overview of the Mars Reconnaissance Orbiter (MRO) science mission, J. Geophys. Res., 112, E05S01, doi:10.1029/2006JE002701.

W. A. Abdou, D. M. Kass, A. Kleinböhl, D. J. McCleese, J. T. Schofield, J. H. Shirley, and R. W. Zurek, Jet Propulsion Laboratory, California Institute of Technology, Pasadena, CA 91109, USA.

J. L. Bandfield, Department of Atmospheric Sciences, University of Washington, Seattle, WA 98195, USA.

S. B. Calcutt, P. G. J. Irwin, P. L. Read, F. W. Taylor, and N. Teanby, Department of Physics, University of Oxford, Oxford OX1 3PU, UK.

N. G. Heavens, Division of Geological and Planetary Sciences, California Institute of Technology, Pasadena, CA 91125, USA.

S. R. Lewis, Department of Physics and Astronomy, Open University, Milton Keynes MK7 6AA, UK.

D. A. Paige, Department of Earth and Space Sciences, University of California, Los Angeles, CA 90095, USA.

M. I. Richardson, Ashima Research, 600 South Lake Ave., Ste. 303, Pasadena, CA 91106, USA. 